ESAIM: M2AN 47 (2013) 1821-1843

DOI: $10.1051 / \mathrm{m} 2 \mathrm{an} / 2013090$
ESAIM: Mathematical Modelling and Numerical Analysis

www.esaim-m2an.org

\title{
A GALERKIN STRATEGY WITH PROPER ORTHOGONAL DECOMPOSITION FOR PARAMETER-DEPENDENT PROBLEMS - ANALYSIS, ASSESSMENTS AND APPLICATIONS TO PARAMETER ESTIMATION
}

\author{
D. Chapelle ${ }^{1}$, A. Gariah ${ }^{2}$, P. Moireau ${ }^{1}$ And J. Sainte-Marie ${ }^{2,3,4}$
}

\begin{abstract}
We address the issue of parameter variations in POD approximations of time-dependent problems, without any specific restriction on the form of parameter dependence. Considering a parabolic model problem, we propose a POD construction strategy allowing us to obtain some a priori error estimates controlled by the POD remainder - in the construction procedure - and some parameterwise interpolation errors for the model solutions. We provide a thorough numerical assessment of this strategy with the FitzHugh-Nagumo 1D model. Finally, we give detailed illustrations of the approach in two parameter estimation applications, the first in a variational estimation framework with the FitzHugh-Nagumo model, and the second with a beating heart mechanical model for which we employ a sequential estimation method to characterize model parameters using real image data in a clinical case.
\end{abstract}

Mathematics Subject Classification. 65M60, 35A35, 35B45, 93E10.

Received May 21, 2012.

Published online October 7, 2013.

\section{INTRODUCTION}

So-called "reduced-order modeling" of partial differential equations (PDEs) is a very active field of research, as exemplified in the "Reduced Basis Method" (see e.g. [22]) and "Proper Orthogonal Decomposition" (POD) $[3,15]$, in particular. In these methods, the order reduction - in the size of the problems to be solved - is typically achieved by using pre-computed solutions to generate a well-chosen subspace within which an approximate solution is then sought, e.g. by Galerkin projection. Of course, since preliminary computations - frequently referred to as "off-line" - are required, this is primarily justified in a "many-query" context, i.e. when many additional solutions need to be computed, as for example when considering parametric variations in the physical coefficients, the loading, the geometry of the system, and so on. Some major motivations for such parametric variations include associated optimization or estimation problems, see e.g. $[2,16]$.

\footnotetext{
Keywords and phrases. Proper Orthogonal Decomposition, parameter variations, estimation, FitzHugh-Nagumo equations, cardiac modeling.

1 Inria Saclay Ile-de-France, Palaiseau, France. dominique.chapelle@inria.fr

2 Inria Paris-Rocquencourt, Le Chesnay, France.

3 Université Paris 6, CNRS UMR 7598, Laboratoire Jacques-Louis Lions, Paris, France.

4 CETMEF, Margny-les-Compiègne, France.
} 
In this paper, we focus on the issue of parameter variations in POD approximations of time-dependent problems, without any specific restriction on the form of parameter dependence. In particular we do not assume this dependence to be linear, as sometimes considered in the literature. The context of parameter variation raises the crucial question of how the POD subspace should be constructed in order to ensure adequate approximation properties for arbitrary values of the parameters, of course within a certain admissible parameter domain to be prescribed. We will analyze this issue in the light of a priori error estimates. Whereas such error estimates are not readily available for POD approximations beyond mere Galerkin-type estimates, some error bounds controlled by the POD remainder - namely, the threshold that can be directly adjusted when originally constructing the POD family - have been recently established in [7]. These new error estimates were obtained under the technical assumption that a given projector be uniformly continuous, which has been numerically verified in various examples, very much in the spirit of the numerical inf-sup test of [6]. However, these estimates were derived in the context of "self-approximation", namely, using the reference solution itself - of which an approximation is sought - to construct the POD family. Of course, in the parameter-dependent framework this strategy is not applicable, and this paper deals with the required extension of analysis. Our analysis will also provide insight into how the POD construction should be adapted, and in particular what specific parameter-instantiated solutions need be generated in the construction process. Note that alternative routes can be considered for handling parameter variations, such as using well-chosen interpolation strategies - between various parameter points - directly on the reduced dynamical operators [1]. Here, we undertake a strictly Galerkin-based approach, and instead use interpolation strategies and estimates as error analysis tools only, and for parameter-dependent solutions themselves.

An outline of the paper is as follows. In the next section, we perform an a priori error analysis for a parameterdependent parabolic model problem and a POD construction strategy - that we refer to as "multi-POD" - by which we concatenate the snapshots of solutions corresponding to given nodes in the parameter space, in order to derive the POD basis. In Section 3, we thus provide a thorough numerical assessment of this strategy with the so-called FitzHugh-Nagumo 1D model, representing biological electrical phenomena by a combination of diffusion and non-linear reaction terms. Finally, in Section 4 we give detailed illustrations of the approach in two parameter estimation applications, the first directly with the previously considered FitzHugh-Nagumo model and a variational estimation problem, and the second with a beating heart mechanical model for which we employ a well-chosen sequential estimation method to characterize some key model parameters using real image data in a clinical case.

\section{Numerical ANALYSis For PARABOliC MODEL PROBLEM}

In order to fix the ideas, we introduce a parabolic parameter-dependent model problem on which we will perform our numerical analysis. We thus consider a governing bilinear form $a$ depending on a parameter vector $\theta \in \Theta \subset \mathbb{R}^{p}$, where $\Theta$ represents an admissible parametric domain - without any particular assumption on the form of the parameter dependence - and the associated evolution problem written in weak form

$$
\left\{\begin{array}{l}
\frac{\mathrm{d}}{\mathrm{d} t}(u(t ; \theta), v)+a(u(t ; \theta), v ; \theta)=(f(t), v), \quad \forall v \in V \\
u(0 ; \theta)=u_{0} .
\end{array}\right.
$$

In many examples, the parameter space will correspond to a simplified description of the spatial variations of some physical parameters, e.g. by considering a - frequently quite coarse - piecewise-constant or piecewiselinear spatial interpolation. In this variational formulation, $V$ denotes the Sobolev space in which we seek the solution, and the scalar product (.,.) should be understood with respect to another less regular Sobolev space $H$ for which we assume we have the compact inclusion $V \subset H$. We use the notations $\|$. $\|$ and $\mid$.| for the norms of $V$ and $H$, respectively. The bilinear form $a$ is taken symmetric, continuous and coercive for any value of $\theta \in \Theta$, with constants

$$
C_{a}(\theta)=\sup _{v, w \neq 0} \frac{a(v, w ; \theta)}{\|v\|\|w\|}, \quad c_{a}(\theta)=\inf _{v \neq 0} \frac{a(v, v ; \theta)}{\|v\|^{2}} .
$$


Then, provided that $f \in L^{2}(0, T ; V)$ and $u_{0} \in H$, there exists a unique solution $u(\theta)$ such that

$$
u(\theta) \in L^{2}(0, T ; V) \cap C([0, T] ; H), \quad \frac{\partial u}{\partial t}(\theta) \in L^{2}\left(0, T ; V^{\prime}\right)
$$

see e.g. [7] and references therein.

Considering now a subspace $V^{l} \subset V$ of finite dimension $l$, the corresponding Galerkin approximation consists in seeking $u^{l}(t ; \theta) \in V^{l}$ such that

$$
\left\{\begin{array}{l}
\frac{\mathrm{d}}{\mathrm{d} t}\left(u^{l}(t ; \theta), v\right)+a\left(u^{l}(t ; \theta), v ; \theta\right)=(f(t), v), \quad \forall v \in V^{l} \\
u^{l}(0 ; \theta)=u_{0}^{l}
\end{array}\right.
$$

with $u_{0}^{l} \in V^{l}$, and we also have a unique solution that satisfies [7]

$$
u^{l}(\theta) \in C\left([0, T] ; V^{l}\right), \quad \frac{\partial u^{l}}{\partial t}(\theta) \in L^{2}\left(0, T ; V^{l}\right)
$$

We denote by $\pi_{V}^{l}$ and $\pi_{H}^{l}$ the orthogonal projectors onto $V^{l}$ with respect to the norms of $V$ and $H$, respectively, and we define as in $[7]$

$$
\sigma_{l}=\left\|\pi_{V}^{l}-\pi_{H}^{l}\right\|_{\mathcal{L}(V)}
$$

Proving that this projection continuity constant is bounded - under some assumptions to be specified - for general POD spaces is an open problem, but when considering specific examples this constant can be numerically computed, using an equivalent eigenproblem in the spirit of the inf-sup test of [6]. In all the examples considered in [7] this constant was found to be numerically bounded.

\subsection{Galerkin error estimates}

We start by adapting the error estimate derived in [7] to take into account the parameter variation. Let us consider $\mathcal{B} \subset \Theta$ a compact subset of the parameter domain for which we assume that the constants

$$
\kappa_{a}(\mathcal{B})=\sup _{\theta \in \mathcal{B}} \frac{C_{a}(\theta)}{c_{a}(\theta)}, \quad c_{a}(\mathcal{B})=\inf _{\theta \in \mathcal{B}} c_{a}(\theta)
$$

are finite and strictly positive. Then, by revisiting the proof of [7], Proposition 7, the following estimate is straightforwardly obtained.

Proposition 2.1. For all $T>0$,

$$
\left\|u-u^{l}\right\|_{C^{0}\left(\mathcal{B} ; L^{2}(0, T ; V)\right)} \leq \frac{1}{\sqrt{c_{a}(\mathcal{B})}}\left|u_{0}^{l}-\pi_{H}^{l} u_{0}\right|+\left(1+\sqrt{\kappa_{a}(\mathcal{B})}\right)\left(1+\sigma_{l}\right)\left\|u-\pi_{V}^{l} u\right\|_{C^{0}\left(\mathcal{B} ; L^{2}(0, T ; V)\right)} .
$$

Of course, in practice it will be extremely difficult to control the uniform approximation error term $\left\|u-\pi_{V}^{l} u\right\|_{C^{0}\left(\mathcal{B} ; L^{2}(0, T ; V)\right)}$ directly from the construction of the Galerkin (POD) subspace, hence we propose an interpolation strategy to circumvent this difficulty.

\subsection{Lagrange interpolation based POD construction and reduction estimates}

From now on, we assume that $\mathcal{B}$ is a $p$-dimensional parameter "box", namely, has the form

$$
\mathcal{B}=\left[a_{1}, b_{1}\right] \times \ldots \times\left[a_{p}, b_{p}\right], \quad a_{i}<b_{i} .
$$


We denote by $Q_{k}$ the usual space of polynomials defined over $\mathbb{R}^{p}$ and of degree at most $k$ in each variable, and we define the standard evenly-spaced Lagrange-interpolation nodes $\left(\theta_{I}\right)_{I \in \mathcal{I}}$ associated with this polynomial space in the box $\mathcal{B}$, with

$$
\mathcal{I}=\left\{I=\left(i_{1}, i_{2}, \ldots, i_{p}\right) \in\{0,1, \ldots, k\}^{p}\right\} .
$$

The corresponding polynomial "shape functions" are denoted by $\left(\lambda_{I}\right)_{I \in \mathcal{I}}$ and satisfy the canonical identity

$$
\lambda_{I}\left(\theta_{J}\right)=\delta_{I J},
$$

with $\delta$ the multi-dimensional Kronecker delta-function. Although interpolation results in this context are rather classical, see e.g. [10], in our case we need to interpolate elements of $L^{2}(0, T ; V)$ instead of reals, which requires a little care to ascertain the validity of the extension. We thus define the interpolation operator

$$
I_{k} w(t ; \theta)=\sum_{I \in \mathcal{I}} \lambda_{I}(\theta) w\left(t ; \theta_{I}\right), \quad \forall w \in C^{0}\left(\mathcal{B} ; L^{2}(0, T ; V)\right),
$$

namely, an endomorphism on $C^{0}\left(\mathcal{B} ; L^{2}(0, T ; V)\right)$, and we will essentially rely on the following classical Lemma [10].

Lemma 2.2. For all $\alpha \in \mathbb{N}^{p}$ such that $|\alpha| \leq k$,

$$
\sum_{I \in \mathcal{I}} \lambda_{I}(\theta)\left(\theta_{I}-\theta\right)^{\alpha} \equiv 0
$$

Then we can establish an approximation bound as follows.

Proposition 2.3. Let $1 \leq r \leq k$ and $w \in C^{r+1}\left(\mathcal{B} ; L^{2}(0, T ; V)\right)$, i.e.

$$
|w|_{C^{r+1}\left(\mathcal{B} ; L^{2}(0, T ; V)\right)}=\sup _{\substack{|\alpha|=r+1 \\ \theta \in \mathcal{B}}}\left\|\partial_{\alpha} w(\theta)\right\|_{L^{2}(0, T ; V)}<\infty .
$$

Then, for all $T>0$,

$$
\begin{aligned}
\left\|w-\pi_{V}^{l} w\right\|_{C^{0}\left(\mathcal{B} ; L^{2}(0, T ; V)\right)} \leq C_{1}(p, k)\left(\mu_{r+1}(\mathcal{B})\right)^{r+1} \mid & \left.w\right|_{C^{r+1}\left(\mathcal{B} ; L^{2}(0, T ; V)\right)} \\
& +C_{2}(p, k)\left\{\sum_{I \in \mathcal{I}}\left\|w\left(\theta_{I}\right)-\pi_{V}^{l} w\left(\theta_{I}\right)\right\|_{L^{2}(0, T ; V)}^{2}\right\}^{1 / 2}
\end{aligned}
$$

with the constants

$$
C_{1}(p, k)=\left\|\sum_{I \in \mathcal{I}}\left|\lambda_{I}\right|\right\|_{C^{0}(\mathcal{B})}, \quad C_{2}(p, k)=\left\|\left\{\sum_{I \in \mathcal{I}}\left|\lambda_{I}\right|^{2}\right\}^{1 / 2}\right\|_{C^{0}(\mathcal{B})}
$$

and the specific measure of $\mathcal{B}$

$$
\mu_{r+1}(\mathcal{B})=\left\{\sum_{|\alpha|=r+1} \frac{\left(b_{1}-a_{1}\right)^{\alpha_{1}}}{\alpha_{1} !} \ldots \frac{\left(b_{p}-a_{p}\right)^{\alpha_{p}}}{\alpha_{p} !}\right\}^{1 /(r+1)}
$$


Proof. Let $p^{l}=w-\pi_{V}^{l} w$. Clearly, for $w \in C^{r+1}\left(\mathcal{B} ; L^{2}(0, T ; V)\right), \pi_{V}^{l} w$ and then $p^{l}$ have regularity $C^{r+1}$ in the parameter. We use the triangle inequality

$$
\left\|p^{l}\right\|_{C^{0}\left(\mathcal{B} ; L^{2}(0, T ; V)\right)} \leq\left\|p^{l}-I_{k} p^{l}\right\|_{C^{0}\left(\mathcal{B} ; L^{2}(0, T ; V)\right)}+\left\|I_{k} p^{l}\right\|_{C^{0}\left(\mathcal{B} ; L^{2}(0, T ; V)\right)} .
$$

We first estimate the interpolation error term. For all $I \in \mathcal{I}$ and all $\theta \in \mathcal{B}, p^{l}$ admits the Taylor expansion

$$
p^{l}\left(\theta_{I}\right)=p^{l}(\theta)+\sum_{s=1}^{r} \sum_{|\alpha|=s} \frac{1}{\alpha !}\left(\theta_{I}-\theta\right)^{\alpha} \partial_{\alpha} p^{l}(\theta)+\sum_{|\alpha|=r+1} \frac{1}{\alpha !}\left(\theta_{I}-\theta\right)^{\alpha} \partial_{\alpha} p^{l}\left(\eta_{I}\left(\theta, \theta_{I}\right)\right),
$$

where $\eta_{I}\left(\theta, \theta_{I}\right) \in\left[\theta, \theta_{I}\right]$. We multiply this expression by $\lambda_{I}(\theta)$ and take the sum for $I \in \mathcal{I}$. Then, by Lemma 2.2 and using $\sum_{I \in \mathcal{I}} \lambda_{I}(\theta) \equiv 1$, this simply becomes

$$
I_{k} p^{l}(\theta)=p^{l}(\theta)+\sum_{|\alpha|=r+1} \frac{1}{\alpha !} \sum_{I \in \mathcal{I}} \lambda_{I}(\theta)\left(\theta_{I}-\theta\right)^{\alpha} \partial_{\alpha} p^{l}\left(\eta_{I}\left(\theta, \theta_{I}\right)\right) .
$$

Taking the $L^{2}(0, T ; V)$ norm and passing to the supremum in $\theta \in \mathcal{B}$ leads to

$$
\left\|p^{l}-I_{k} p^{l}\right\|_{C^{0}\left(\mathcal{B} ; L^{2}(0, T ; V)\right)} \leq C_{1}(p, k)\left(\mu_{r+1}(\mathcal{B})\right)^{r+1}\left|p^{l}\right|_{C^{r+1}\left(\mathcal{B} ; L^{2}(0, T ; V)\right)},
$$

and we also remark that $\left|p^{l}\right|_{C^{r+1}\left(\mathcal{B} ; L^{2}(0, T ; V)\right)} \leq|w|_{C^{r+1}\left(\mathcal{B} ; L^{2}(0, T ; V)\right)}$. Then, taking the $L^{2}(0, T ; V)$ norm on the interpolation formula (2.4), we estimate the second term in (2.6) by a function of the grid evaluations only. Finally, by a Cauchy-Schwarz inequality,

$$
\left\|I_{k} p^{l}\right\|_{C^{0}\left(\mathcal{B} ; L^{2}(0, T ; V)\right)} \leq C_{2}(p, k)\left\{\sum_{I \in \mathcal{I}}\left\|p^{l}\left(\theta_{I}\right)\right\|_{L^{2}(0, T ; V)}^{2}\right\}^{1 / 2}
$$

which concludes our proof.

Now, substituting (2.5) into (2.3), we see that we are naturally led to considering the minimization problem

$$
\min _{\tilde{\pi}_{V}^{l}} \sum_{I \in \mathcal{I}}\left\|u\left(\theta_{I}\right)-\tilde{\pi}_{V}^{l} u\left(\theta_{I}\right)\right\|_{L^{2}(0, T ; V)}^{2}
$$

in the construction of the Galerkin subspace. This should be understood as a minimization over all possible finite-dimensional subspaces of dimension $l$, and we recognize a rather simple extension of the criterion prevailing in standard POD definitions. Hence, introducing $\widehat{\operatorname{Cov}} \in \mathcal{L}(V)$ the multi-covariance operator - thus called by extension of the standard terminology used in principal component analysis concerning the empirical covariance operator associated with the data, here the collection of solutions $u\left(\theta_{I}\right)$ - defined by

$$
\widehat{\operatorname{Cov}} \varphi=\sum_{I \in \mathcal{I}} \int_{0}^{T}\left(u\left(\theta_{I}\right)(t), \varphi\right)_{V} u\left(\theta_{I}\right)(t) \mathrm{d} t
$$

with $(., .)_{V}$ denoting the scalar product of $V$, we give the result without proof, see [7] and references therein for details.

Proposition 2.4. There exists a unique sequence $\left(\omega_{i}\right)_{i \in L}$ - with L either finite or infinite - of strictly positive reals $\omega_{i}$ such that

$$
\begin{gathered}
\omega_{1} \geq \omega_{2} \geq \ldots \geq \omega_{N} \quad \text { if } L \text { finite } \quad(L=\{1,2, \ldots, N\}), \\
\omega_{1} \geq \omega_{2} \geq \ldots \geq \omega_{i} \geq \ldots, \quad \omega_{i} \underset{i \rightarrow \infty}{\longrightarrow} 0 \quad \text { if } L \text { infinite } \quad\left(L=\mathbb{N}^{*}\right),
\end{gathered}
$$


and an orthonormal sequence $\left(\varphi_{i}\right)_{i \in I}$ of $V$ of associated eigenvectors of $\widehat{\operatorname{Cov}}$, in finite number for each non-zero eigenvalue,

$$
\widehat{\operatorname{Cov}} \varphi_{i}=\omega_{i} \varphi_{i}, \quad \forall i \in L,
$$

such that $\left(\varphi_{i}\right)_{i \in L}$ is total in the orthogonal complement of the kernel of $\widehat{\operatorname{Cov}}$, namely,

$$
V=\operatorname{Ker} \widehat{\operatorname{Cov}} \stackrel{\perp}{\oplus} \overline{\operatorname{Span}\left\{\varphi_{i}\right\}_{i \in I}} .
$$

Then, for all $l \in L$, a solution $\pi_{V}^{l}$ of Problem (2.7) is given by

$$
\operatorname{Im} \pi_{V}^{l}=\operatorname{Span}\left(\varphi_{1}, \ldots, \varphi_{l}\right) .
$$

Moreover, $\left(\omega_{i}\right)_{i \in L}$ is the only sequence such that the minimum value verifies

$$
\sum_{I \in \mathcal{I}}\left\|u\left(\theta_{I}\right)-\pi_{V}^{l} u\left(\theta_{I}\right)\right\|_{L^{2}(0, T ; V)}^{2}=\min _{\tilde{\pi}_{V}^{l}} \sum_{I \in \mathcal{I}}\left\|u\left(\theta_{I}\right)-\tilde{\pi}_{V}^{l} u\left(\theta_{I}\right)\right\|_{L^{2}(0, T ; V)}^{2}=\sum_{i>l} \omega_{i} .
$$

Note that, in practice, the time integral in the criterion (2.7) will be approximated with a given time sampling corresponding to series of solution "snapshots". The actual computation of the POD vectors can then be performed by using the standard algorithm in which the snapshots associated with all parametrized solutions $\left(u\left(\theta_{I}\right)\right)_{I \in \mathcal{I}}$ are simply concatenated. For this reason, we will refer to our approach as a "multi-POD" strategy.

Finally, defining

$$
\varepsilon(l)=\left\{\sum_{i>l} \omega_{i}\right\}^{\frac{1}{2}},
$$

we can summarize our reduction estimate in the following proposition.

Proposition 2.5. Assume that $V^{l}$ is the minimizer of (2.7). Then, for all $T>0$ and any $1 \leq r \leq k$,

$$
\begin{aligned}
\left\|u-u^{l}\right\|_{C^{0}\left(\mathcal{B} ; L^{2}(0, T ; V)\right)} \leq \frac{1}{\sqrt{c_{a}(\mathcal{B})}} \mid u_{0}^{l}-\pi_{H}^{l} & u_{0} \mid+\left(1+\sqrt{\kappa_{a}(\mathcal{B})}\right)\left(1+\sigma_{l}\right) \\
& \times\left(C_{1}(p, k)\left(\mu_{r+1}(\mathcal{B})\right)^{r+1}|u|_{C^{r+1}\left(\mathcal{B} ; L^{2}(0, T ; V)\right)}+C_{2}(p, k) \varepsilon(l)\right) .
\end{aligned}
$$

From this error estimate, it is very clear as to how we can control the reduction error by first adjusting the size of the parameter box and/or the interpolation degree, then by selecting the adequate size for the POD family. Note that in practice this induces quite drastic limitations as to the number of parameters that can be considered, since the number of solutions to be generated - hence, also the size of the matrix on which the singular-value decomposition is performed to compute the POD basis - increases exponentially with the dimension of the parameter space. Nevertheless, of course these computations are all carried out off-line and the direct simulations themselves can be obtained in parallel, while some optimization can be performed in the interpolation strategy, see also Section 5.

\section{Numerical assessments for FitzHugh-Nagumo model}

\subsection{Model presentation and discretization}

Rather than providing a simple numerical verification of our above error estimates, we will consider a slightly more complex model equation, namely, the one-dimensional so-called FitzHugh-Nagumo model $[11,20]$ - originally devised to represent electrical phenomena in an axon by a combination of diffusion and non-linear reaction terms - which reads in variational form

$$
\frac{\mathrm{d}}{\mathrm{d} t}(u(t), v)+a(u(t), v ; \theta)=(f(u(t))-\gamma w(t), v), \quad \forall v \in V,
$$


coupled with the ordinary differential equation

$$
\frac{\partial w}{\partial t}=\alpha u-\beta w
$$

with initial conditions

$$
u(0)=u_{0}, \quad w(0)=0 .
$$

In this system we have

$$
a(u, v ; \theta)=\sum_{q=1}^{p} \theta^{(q)} \int_{\Omega_{q}} u^{\prime} v^{\prime} d x,
$$

the diffusion term with several piecewise-constant values of the diffusion parameter, while the reaction term is based on the cubic polynomial

$$
f(u)=-f_{0} u(u-1)\left(u-f_{1}\right),
$$

and all the other unspecified quantities in (3.1)-(3.4) denote constants to be prescribed. The problem is posed in the segment $x \in[0,1]$, with homogeneous Dirichlet boundary conditions, hence we consider $V=H_{0}^{1}([0,1])$, whereas $H=L^{2}([0,1])$.

We discretize the problem space-wise with a standard $P_{1}$-Lagrange finite element procedure based on an even subdivision of $[0,1]$ into $N$ elements, and time-wise with a theta-method and a constant time step $\Delta t$. Namely, the totally discrete solution $\left(u_{h}^{n}, w_{h}^{n}\right)$ - meant to approximate $(u(n \Delta t), w(n \Delta t))$ - satisfies the system

$$
\left\{\begin{aligned}
\left(\frac{u_{h}^{n+1}-u_{h}^{n}}{\Delta t}, v_{h}\right)+ & a\left(\eta u_{h}^{n+1}+(1-\eta) u_{h}^{n}, v_{h} ; \theta\right) \\
& =\eta\left(f\left(u_{h}^{n+1}\right)-\gamma w_{h}^{n+1}, v_{h}\right)+(1-\eta)\left(f\left(u_{h}^{n}\right)-\gamma w_{h}^{n}, v_{h}\right), \quad \forall v_{h} \in V_{h} \\
\frac{w_{h}^{n+1}-w_{h}^{n}}{\Delta t}=\eta\left(\alpha u_{h}^{n+1}-\beta w_{h}^{n+1}\right)+(1-\eta)\left(\alpha u_{h}^{n}-\beta w_{h}^{n}\right) &
\end{aligned}\right.
$$

with $V_{h}$ the space of continuous piecewise-linear functions vanishing in 0 and 1 , and $\eta$ the parameter of the theta-method (thus-denoted to be distinguished from the physical parameter $\theta$ ). Of course, this gives an implicit non-linear system in $\left(u_{h}^{n+1}, w_{h}^{n+1}\right)$, which we solve for using a Newton-type algorithm.

Then, the reduced-order model consists in seeking $\left(u^{l, n}, w^{l, n}\right)$ with $u^{l, n}$ in $V^{l}$-an adequate Galerkin subspace of the discrete space $V_{h}$ - and satisfying

$$
\left\{\begin{aligned}
\left(\frac{u^{l, n+1}-u^{l, n}}{\Delta t}, v\right)+a\left(\eta u^{l, n+1}+(1-\eta) u^{l, n}, v ; \theta\right) & =\eta\left(f\left(u^{l, n+1}\right)-\gamma w^{l, n+1}, v\right)+(1-\eta)\left(f\left(u^{l, n}\right)-\gamma w^{l, n}, v\right), \quad \forall v \in V^{l} \\
\frac{w^{l, n+1}-w^{l, n}}{\Delta t}= & \eta\left(\alpha u^{l, n+1}-\beta w^{l, n+1}\right)+(1-\eta)\left(\alpha u^{l, n}-\beta w^{l, n}\right)
\end{aligned}\right.
$$

Our objective in the numerical assessments will be to study the reduction error

$$
\left\|u_{h}-u^{l}\right\|_{L_{\Delta t}^{2}(0, T ; V)}=\left\{\sum_{n=0}^{N_{T}}\left\|u_{h}^{n}-u^{l, n}\right\|_{V}^{2} \Delta t\right\}^{\frac{1}{2}}
$$

when varying the parameter vector $\theta$ throughout the box $\mathcal{B}$ for a fixed Galerkin subspace $V^{l}$.

\subsection{Assessment description and results}

In the following assessments, the parameter space will map two piecewise-constant values of the diffusion coefficient associated with each of the two halves of the interval $[0,1]$, and we will consider a rather large 
TABLE 1. Modeling constants.

\begin{tabular}{|lccc|}
\hline $\bar{\theta}$ & $f(u)$ & $u_{0}$ & $(\alpha, \beta, \gamma)$ \\
\hline $2 \times 10^{-3}$ & $f_{0}=20, f_{1}=0.1$ & $A=1, m=0.5, \sigma=0.25$ & $(0.05,0.01,20)$ \\
\hline
\end{tabular}

TABLE 2. Discretization constants.

\begin{tabular}{|lccc|}
\hline$N$ & $N_{T}$ & $\Delta t$ & $\eta$ \\
\hline 200 & 200 & $2.5 \times 10^{-2}$ & $2 / 3$ \\
\hline
\end{tabular}

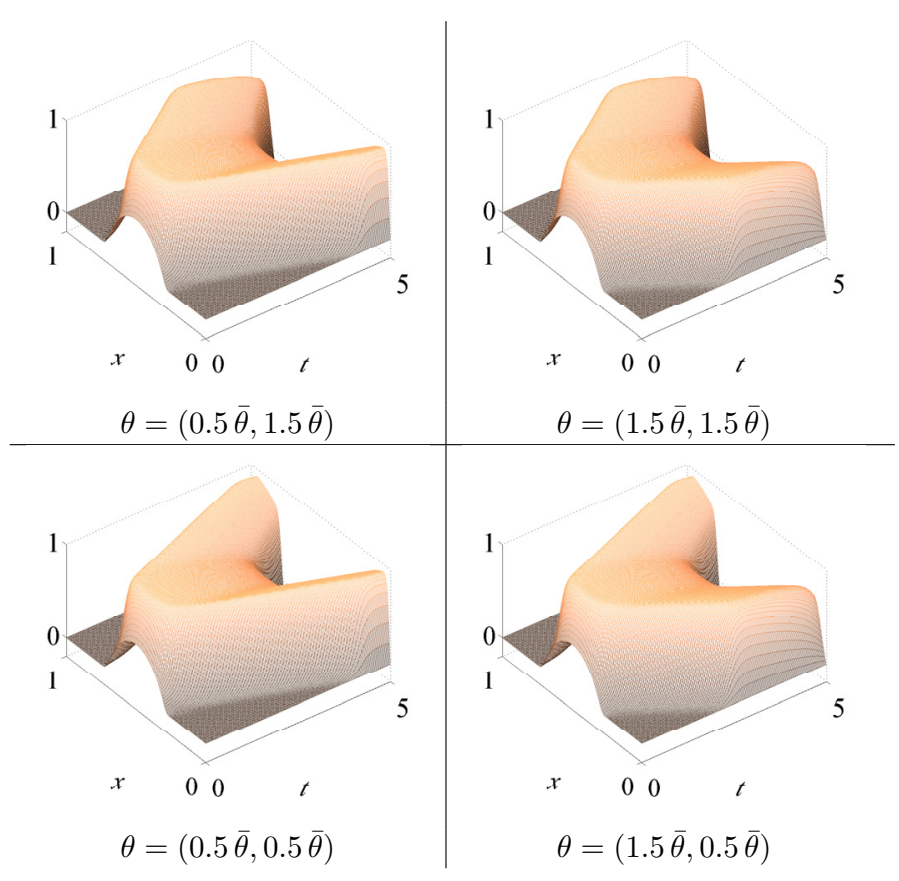

FiguRE 1. Reference solutions obtained for parameter box vertices in FitzHugh-Nagumo model.

parameter box of the type $\mathcal{B}=[0.5 \bar{\theta}, 1.5 \bar{\theta}]^{2}$. As initial condition, we will set smooth "pulses" defined by

$$
u_{0}(x)= \begin{cases}\frac{A}{2} \exp \left(1-\frac{1}{1-\left(\frac{x-m}{\sigma}\right)^{2}}\right) & \text { if }|x-m|<\sigma \\ 0 & \text { otherwise. }\end{cases}
$$

As our initial interpolation strategy will rely on simple $Q_{1}$ bilinear polynomials in the whole box $\mathcal{B}$, we show in Figure 1 the reference solutions obtained for each of the 4 parameter vertices with rather fine discretization parameters - see Table 2, and also Table 1 for modeling constant definitions. We clearly observe the wellknown propagative nature of the solution, and how the diffusion parameter directly conditions the propagation velocity. These four different solutions are also used to compute the multi-POD vectors as explained in the previous section, with the same time sampling as the computational time step $\Delta t$.

We will then compare three error indicators as functions of the POD rank $l$-i.e. the number of POD vectors used in the Galerkin approximation - namely, 

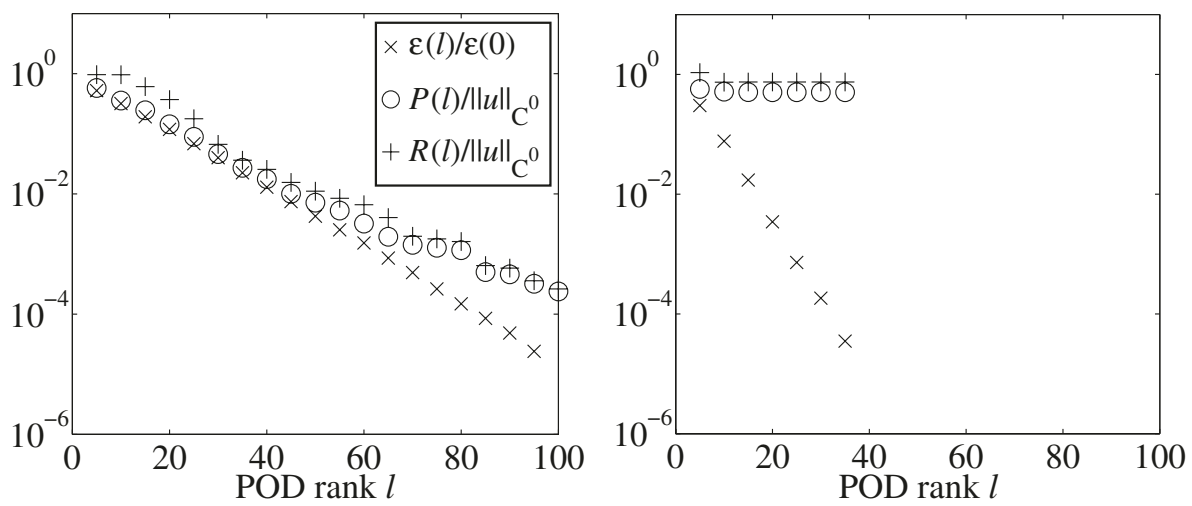

Figure 2. Error indicators vs. POD rank for multi-POD solutions (left) compared to standard POD reduction (right).

- the maximum reduction error (for all parameter values)

$$
R(l)=\left\|u_{h}(\theta)-u^{l}(\theta)\right\|_{C^{0}\left(\mathcal{B} ; L_{\Delta t}^{2}(0, T ; V)\right)},
$$

- the maximum projection error

$$
P(l)=\left\|u_{h}(\theta)-\pi_{V}^{l} u_{h}(\theta)\right\|_{C^{0}\left(\mathcal{B} ; L_{\Delta t}^{2}(0, T ; V)\right)},
$$

- and the POD remainder $\varepsilon(l)$.

For practical purposes, the supremum operation - over the parameter box - included in the computation of $R(l)$ and $P(l)$ will be approximated by taking the maximum value on a Lagrangian grid of $6 \times 6$ parameter "nodes".

We show the computed error indicators in Figure 2, compared with similar indicators obtained when using the "naive" approach of constructing a standard POD set based on the single solution associated with the center of the parameter box, namely, $\theta=(\bar{\theta}, \bar{\theta})$. We observe a very good overall performance of the multi-POD strategy with a maximum relative reduction error of $1 \%$ for about 45 POD vectors. Moreover, reduction and projection errors are very close, which shows that a numerical behavior of the type established in Proposition 2.1 also prevails here, even though the system considered lies beyond the scope of our above numerical analysis. Likewise, while we have not proven that the estimate (2.8) of Proposition 2.5 holds in this case, our assessment results are in good numerical agreement with this estimate since the reduction (and projection) errors closely follow the POD remainder decrease in relative values, in fact down to unexpectedly small orders of magnitude considering the size of the parameter box and the coarseness of our interpolation strategy. By contrast, the reduced solutions computed with a standard POD strategy hardly display any convergence - see Figure 2 (right). This behavior is clearly due to a very poor approximation capability of this POD family when varying the parameters, since $P(l)$ and $R(l)$ are very close in this case too, and yet plotting the first vectors of the two POD families does not show any striking qualitative difference, see Figure 3. This emphasizes that convergence is not easily achieved in Galerkin approximations for this type of system presenting propagative solutions with varying velocities.

In fact, an even subtler aspect of the FitzHugh-Nagumo model is that it can also have solutions of essentially diffusive character, depending on the amplitude of the initial condition. We thus now choose a smaller amplitude for the initial pulse, with a value $A=A_{c}=0.267$ adjusted so that we have both propagative and diffusive solutions in the parameter box considered. The corresponding error indicators are displayed in Figure 4, where we see that the multi-POD performance has drastically deteriorated, and is now hardly better than that of 

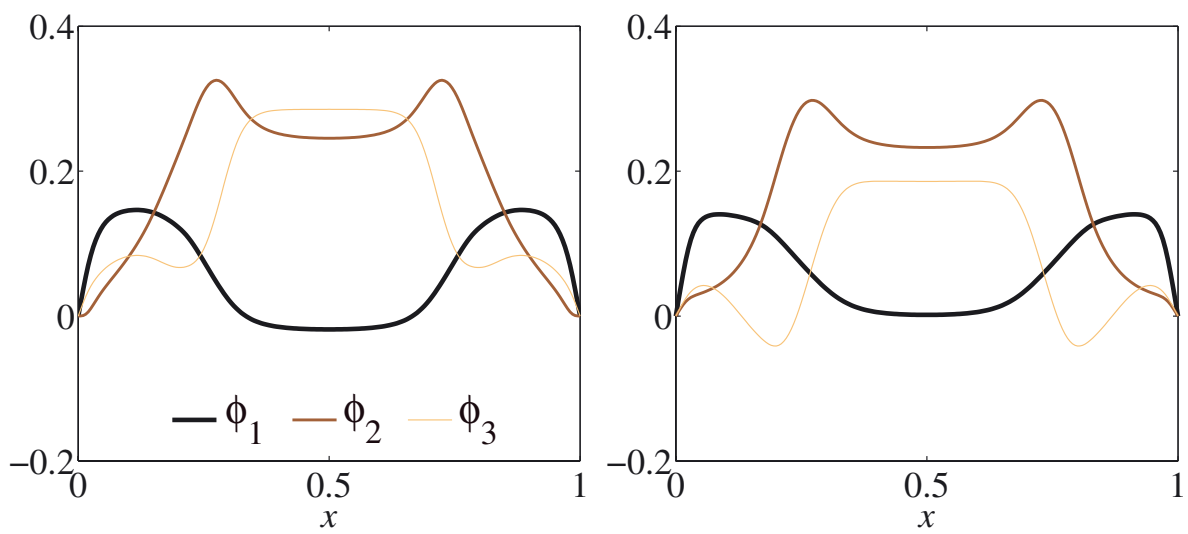

Figure 3. First 3 POD modes for multi-POD (left) vs. standard (right) strategies.
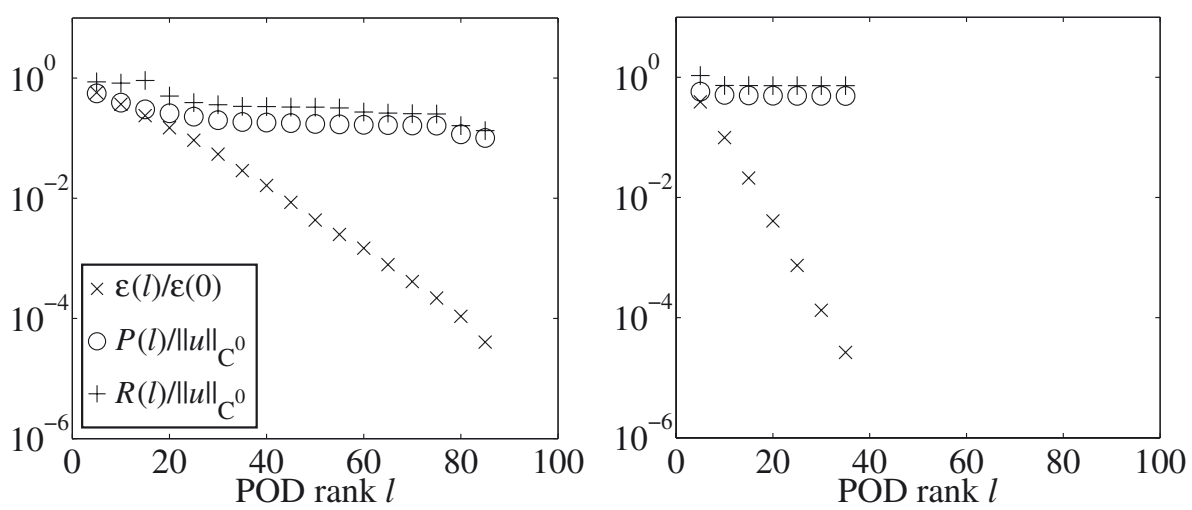

Figure 4. Error indicators vs. POD rank for multi-POD solutions at critical amplitude $A_{c}$ (left) compared to standard POD reduction (right).

the standard POD reduction, indeed. However, it is quite natural to conjecture that this is due to an increased interpolation error - recall (2.8) - as the parameter box is very large, and furthermore now contains dramatically different solutions. In order to reduce the interpolation error we can then either reduce the box size or increase the interpolation order. The corresponding assessment results are shown in Figure 5, where the much improved performance of the multi-POD strategy appears to corroborate our conjecture ${ }^{5}$.

\section{Applications to parameter estimation}

Recalling that our major motivation for considering parameter variations with POD reduction is to devise suitable reduction strategies for estimation problems, we now present two different estimation applications, namely, first variational estimation for the above-presented FitzHugh-Nagumo model, and finally a sequential estimation problem for a mechanical beating heart model for which the data considered consist of real-life clinical measurements, in particular with Magnetic Resonance (MR) image sequences.

\footnotetext{
${ }^{5}$ The reduced box still contains both propagative and diffusive solutions.
} 

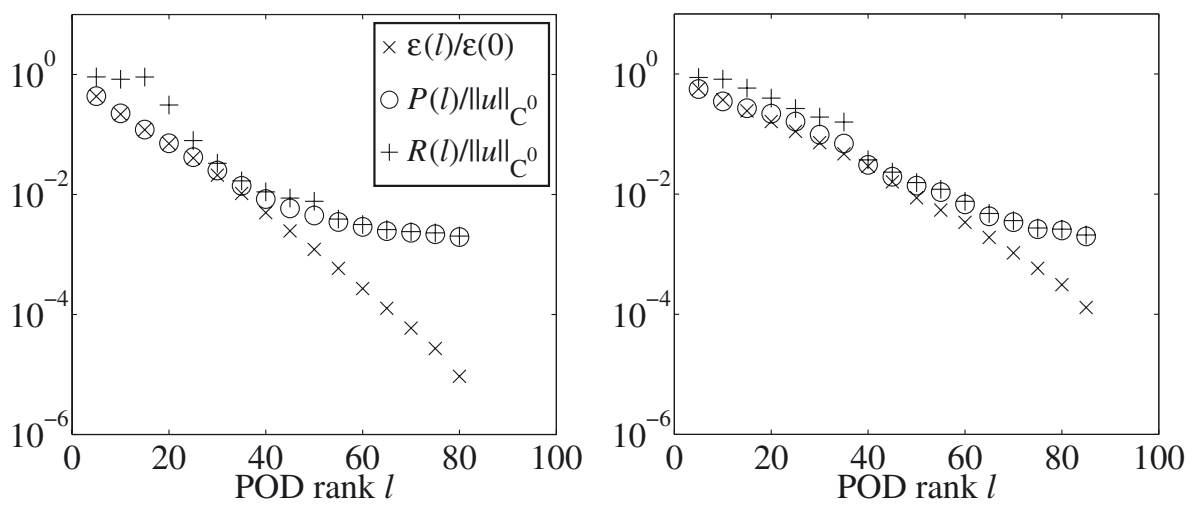

FiguRe 5. Error indicators vs. POD rank for multi-POD at critical amplitude $A_{c}$ in reduced box $\mathcal{B}=[0.875 \bar{\theta}, 1.125 \bar{\theta}]^{2}$ (left) and for $Q_{2}$ multi-POD in full box (right).

\subsection{Variational estimation for FitzHugh-Nagumo model}

Even though we are primarily interested in parameter estimation, it is generally sound to formulate a joint state-parameter estimation problem - namely, in which the initial condition is also estimated - in order to ensure that the estimation procedure is robust with respect to uncertainties, or even numerical inaccuracies, in this initial condition. Nevertheless, for practical purposes concerning the convergence of the gradient-based minimization algorithm, we introduce a reduced parametrization of the initial condition in the form (at the discrete level)

$$
u_{h}^{0}=\sum_{i=1}^{N_{\text {modes }}} \xi^{(i)} \psi_{i}
$$

where the discrete fields $\left(\psi_{i}\right)$ will be here chosen as the eigenmodes of the discrete Laplace operator, order as usual by increasing associated eigenvalue. We then introduce the following discrete estimation criterion

$$
J_{\Delta t}(\xi, \theta)=\frac{1}{2}\left\|\xi-\xi_{0}\right\|_{Q_{\xi}}^{2}+\frac{1}{2}\left\|\theta-\theta_{0}\right\|_{Q_{\theta}}^{2}+\frac{1}{2} \sum_{n=0}^{N_{T}-1}\left\|Z(n \Delta t)-H u_{h}^{n}(\xi, \theta)\right\|_{Q_{Z}}^{2} \Delta t,
$$

where $Z$ denotes a finite-dimensional vector of available measurements given by

$$
Z(t)=H u(t)
$$

with $u$ the reference solution and $H$ the so-called observation operator - assumed to be linear here. The norms denoted by the symbols $Q_{\xi}, Q_{\theta}$ and $Q_{Z}$ need to be adequately prescribed depending on the a priori knowledge on the uncertainty prevailing for each corresponding quantity, and on the estimation objectives. Note that we directly formulate a discrete criterion associated with the discrete problem - although the consistent relation with a continuous criterion is immediate to see - as is classical in numerical optimization to obtain crucial mathematical properties, rather than discretizing the adjoint problem corresponding to the continuous criterion, see e.g. [9].

The estimation problem then consists in seeking the minimization of the criterion $J_{\Delta t}$. This is typically handled by gradient-based descent algorithms in which the gradient is computed via the solution of the so-called 
adjoint problem, which in our case reads

$$
\left\{\begin{array}{l}
\left(\frac{p_{h}^{n+1}-p_{h}^{n}}{\Delta t}, v_{h}\right)-a\left(\eta p_{h}^{n}+(1-\eta) p_{h}^{n+1}, v_{h} ; \theta\right)+\left(\eta p_{h}^{n}+(1-\eta) p_{h}^{n+1}, d_{u} f\left(u_{h}^{n}\right) \cdot v_{h}\right) \\
+\alpha\left(\eta s_{h}^{n}+(1-\eta) s_{h}^{n+1}, v_{h}\right)=-\left(Z(n \Delta t)-H u_{h}^{n}(\xi, \theta), H v_{h}\right)_{Q_{Z}}, \quad \forall v_{h} \in V_{h} \\
\frac{s_{h}^{n+1}-s_{h}^{n}}{\Delta t}-\eta\left(\gamma p_{h}^{n}+\beta s_{h}^{n}\right)-(1-\eta)\left(\gamma p_{h}^{n+1}+\beta s_{h}^{n+1}\right)=0
\end{array}\right.
$$

where $p_{h}^{n}$ and $s_{h}^{n}$ are the adjoint variables associated with $u_{h}^{n}$ and $w_{h}^{n}$, respectively, satisfying the final conditions

$$
p_{h}^{N_{T}}=0, \quad s_{h}^{N_{T}}=0 .
$$

We then have the following result concerning the gradient of the criterion.

Proposition 4.1. For any $(\delta \xi, \delta \theta) \in \mathbb{R}^{N_{\text {modes }}} \times \mathbb{R}^{p}$, defining

$$
\delta u_{h}^{0}=\sum_{i=1}^{N_{\text {modes }}} \delta \xi^{(i)} \psi_{i}
$$

we have the identity

$$
\begin{aligned}
d_{(\xi, \theta)} J_{\Delta t} \cdot(\delta \xi, \delta \theta)=\left(\xi-\xi_{0}\right) \cdot Q_{\xi} \cdot \delta \xi+\left(\theta-\theta_{0}\right) \cdot Q_{\theta} \cdot \delta \theta-\left(p_{h}^{0}, \delta u_{h}^{0}\right) & \\
& +\sum_{n=0}^{N_{T}-1} \Delta t \frac{\partial a}{\partial \theta}\left(\eta u_{h}^{n+1}+(1-\eta) u_{h}^{n}, p_{h}^{n+1} ; \theta\right) \cdot \delta \theta \\
& \quad+\eta \Delta t\left[-a\left(\delta u_{h}^{0}, p_{h}^{0} ; \theta\right)+\left(d_{u} f\left(u_{h}^{0}\right) \cdot \delta u_{h}^{0}, p_{h}^{0}\right)+\alpha\left(s_{h}^{0}, \delta u_{h}^{0}\right)\right] .
\end{aligned}
$$

Proof. We sketch the proof, as it is not entirely standard due to the coupled system in a discrete time setting. Using the natural compact notation

$$
\delta u_{h}^{n}=d_{(\xi, \theta)} u_{h}^{n} \cdot(\delta \xi, \delta \theta), \quad \delta w_{h}^{n}=d_{(\xi, \theta)} w_{h}^{n} \cdot(\delta \xi, \delta \theta),
$$

by differentiating (3.5) we obtain

$$
\left\{\begin{aligned}
\left(\frac{\delta u_{h}^{n+1}-\delta u_{h}^{n}}{\Delta t}, v_{h}\right)+a\left(\eta \delta u_{h}^{n+1}+(1-\eta) \delta u_{h}^{n}, v_{h} ; \theta\right) & \\
+\frac{\partial a}{\partial \theta}\left(\eta u_{h}^{n+1}+(1-\eta) u_{h}^{n}, v_{h} ; \theta\right) & \cdot \delta \theta=\eta\left(d_{u} f\left(u_{h}^{n+1}\right) \cdot \delta u_{h}^{n+1}-\gamma \delta w_{h}^{n+1}, v_{h}\right) \\
& +(1-\eta)\left(d_{u} f\left(u_{h}^{n}\right) \cdot \delta u_{h}^{n}-\gamma \delta w_{h}^{n}, v_{h}\right), \quad \forall v_{h} \in V_{h} \\
\frac{\delta w_{h}^{n+1}-\delta w_{h}^{n}}{\Delta t}=\eta\left(\alpha \delta u_{h}^{n+1}-\beta \delta w_{h}^{n+1}\right) & +(1-\eta)\left(\alpha \delta u_{h}^{n}-\beta \delta w_{h}^{n}\right)
\end{aligned}\right.
$$

with $\delta u_{h}^{0}$ as in (4.3) and $\delta w_{h}^{n}=0$. Of course, the main difficulty lies in computing the derivative of the last term in (4.1), viz.

$$
-\sum_{n=0}^{N_{T}-1} \Delta t\left(Z(n \Delta t)-H u_{h}^{n}(\xi, \theta), H \delta u_{h}^{n}\right)_{Q_{Z}}
$$

To that purpose, we use the first equation of the adjoint problem (4.2), choosing $\delta u_{h}^{n}$ as test function and summing over $n$. We are thus led to handling the term

$$
\sum_{n=0}^{N_{T}-1} \Delta t\left(\frac{p_{h}^{n+1}-p_{h}^{n}}{\Delta t}, \delta u_{h}^{n}\right)=-\sum_{n=0}^{N_{T}-1} \Delta t\left(p_{h}^{n+1}, \frac{\delta u_{h}^{n+1}-\delta u_{h}^{n}}{\Delta t}\right)-\left(p_{h}^{0}, \delta u_{h}^{0}\right) .
$$


Combined with the first equation of (4.5) with $p_{h}^{n+1}$ as a test function, we get

$$
\begin{aligned}
& -\sum_{n=0}^{N_{T}-1} \Delta t\left(Z(n \Delta t)-H u_{h}^{n}(\xi, \theta), H \delta u_{h}^{n}\right)_{Q_{Z}} \\
& \sum_{n=0}^{N_{T}-1} \Delta t\left\{\frac{\partial a}{\partial \theta}\left(\eta u_{h}^{n+1}+(1-\eta) u_{h}^{n}, p_{h}^{n+1} ; \theta\right) \cdot \delta \theta+\alpha\left(\eta s_{h}^{n}+(1-\eta) s_{h}^{n+1}, \delta u_{h}^{n}\right)\right. \\
& \left.\quad+\gamma\left(\eta p_{h}^{n}+(1-\eta) p_{h}^{n+1}, \delta w_{h}^{n}\right)\right\}+\eta \Delta t\left[-a\left(\delta u_{h}^{0}, p_{h}^{0} ; \theta\right)+\left(d_{u} f\left(u_{h}^{0}\right) \cdot \delta u_{h}^{0}, p_{h}^{0}\right)\right] .
\end{aligned}
$$

To simplify this expression, we need to now use the second equation of the adjoint problem (4.2) with $\delta w_{h}^{n}$ as test function, combined with the second equation of (4.5) with $s_{h}^{n+1}$ as a test function, which gives

$$
\sum_{n=0}^{N_{T}-1} \Delta t\left\{\alpha\left(\eta s_{h}^{n}+(1-\eta) s_{h}^{n+1}, \delta u_{h}^{n}\right)+\gamma\left(\eta p_{h}^{n}+(1-\eta) p_{h}^{n+1}, \delta w_{h}^{n}\right)\right\}=\eta \alpha \Delta t\left(s_{h}^{0}, \delta u_{h}^{0}\right) .
$$

The final expression of (4.4) is then straightforward.

Note that we have obtained the expression of the criterion gradient for a general dependence of the bilinear form $a$ with respect to the parameter vector $\theta$, but since in our specific case we have a linear relation the derivative is easily computed.

We can now define the reduced estimation problem which consists in seeking the minimum of the criterion

$$
J_{\Delta t}^{l}(\xi, \theta)=\frac{1}{2}\left\|\xi-\xi_{0}\right\|_{Q_{\xi}}^{2}+\frac{1}{2}\left\|\theta-\theta_{0}\right\|_{Q_{\theta}}^{2}+\frac{1}{2} \sum_{n=0}^{N_{T}-1}\left\|Z(n \Delta t)-H u^{l, n}(\xi, \theta)\right\|_{Q_{Z}}^{2} \Delta t,
$$

for $u^{l, n}(\xi, \theta) \in V^{l}$ solution of (3.6) with

$$
u^{l, 0}=\pi_{V}^{l}\left(\sum_{i=1}^{N_{\text {modes }}} \xi^{(i)} \psi_{i}\right) .
$$

It is then very straightforward to adapt the adjoint problem into

$$
\left\{\begin{array}{l}
\left(\frac{p^{l, n+1}-p^{l, n}}{\Delta t}, v\right)-a\left(\eta p^{l, n}+(1-\eta) p^{l, n+1}, v ; \theta\right)+\left(\eta p^{l, n}+(1-\eta) p^{l, n+1}, d_{u} f\left(u^{l, n}\right) \cdot v\right) \\
+\alpha\left(\eta s^{l, n}+(1-\eta) s^{l, n+1}, v\right)=-\left(Z(n \Delta t)-H u^{l, n}(\xi, \theta), H v\right)_{Q_{Z}}, \quad \forall v \in V^{l} \\
\frac{s^{l, n+1}-s^{l, n}}{\Delta t}-\eta\left(\gamma p^{l, n}+\beta s^{l, n}\right)-(1-\eta)\left(\gamma p^{l, n+1}+\beta s^{l, n+1}\right)=0
\end{array}\right.
$$

with final conditions $p^{l, N_{T}}=s^{l, N_{T}}=0$, and we infer the following expression of the criterion gradient.

Proposition 4.2. For any $(\delta \xi, \delta \theta) \in \mathbb{R}^{N_{\text {modes }}} \times \mathbb{R}^{p}$, defining

$$
\delta u^{l, 0}=\pi_{V}^{l}\left(\sum_{i=1}^{N_{\text {modes }}} \delta \xi^{(i)} \psi_{i}\right)
$$

we have the identity

$$
\begin{aligned}
d_{(\xi, \theta)} J_{\Delta t}^{l} \cdot(\delta \xi, \delta \theta)=\left(\xi-\xi_{0}\right) \cdot & Q_{\xi} \cdot \delta \xi+\left(\theta-\theta_{0}\right) \cdot Q_{\theta} \cdot \delta \theta-\left(p^{l, 0}, \delta u^{l, 0}\right) \\
& +\sum_{n=0}^{N_{T}-1} \Delta t \\
\quad & \frac{\partial a}{\partial \theta}\left(\eta u^{l, n+1}+(1-\eta) u^{l, n}, p^{l, n+1} ; \theta\right) \cdot \delta \theta \\
& \quad+\eta \Delta t\left[-a\left(\delta u^{l, 0}, p^{l, 0} ; \theta\right)+\left(d_{u} f\left(u^{l, 0}\right) \cdot \delta u^{l, 0}, p^{l, 0}\right)+\alpha\left(s^{l, 0}, \delta u^{l, 0}\right)\right] .
\end{aligned}
$$


TABLE 3. Estimation constants.

\begin{tabular}{|cccccccc|}
\hline$\xi_{0}$ & $N_{\text {modes }}$ & $Q_{\xi}$ & $\theta_{0}$ & $\theta_{\text {ref }}$ & $Q_{\theta}$ & $\sigma_{\chi}$ & $Q_{Z}$ \\
\hline$\xi_{\text {ref }}$ & 10 & $4 \times 10^{4} \operatorname{Id}_{10}$ & $(1,1) \bar{\theta}$ & $(0.55,0.7) \bar{\theta}$ & $4 / \bar{\theta}^{2} \operatorname{Id}_{2}$ & $2 \times 10^{-3} / \sqrt{\Delta t}$ & $1 /\left(\sigma_{\chi}^{2} \Delta t\right) \operatorname{Id}_{9}$ \\
\hline
\end{tabular}

TABLE 4. Reference estimation errors.

\begin{tabular}{|lcc|}
\hline & $A=1$ & $A=A_{c}$ \\
\hline Iteration \# & 14 & 61 \\
$\tau_{\xi}(\%)$ & 0.13 & 0.94 \\
$\tau_{\theta}(\%)$ & 0.16 & 0.25 \\
$\tau_{u}(\%)$ & 0.32 & 0.49 \\
\hline
\end{tabular}

In fact, we have to deal with an additional difficulty, namely, that the diffusion parameters should be restricted to strictly positive values in order for the bilinear form $a$ to remain positive. In order to enforce this constraint, we reparametrize the problem in the form

$$
\theta^{(q)}=\mathrm{e}^{v^{(q)}}, \quad 1 \leq q \leq p,
$$

and minimize the criterion with respect to these reparametrized variables, with straightforward expressions of the gradient obtained by the chain rule.

We proceed by presenting an assessment of the reduced estimation for the proposed multi-POD strategy. To that purpose we choose some given parameter values $\theta_{\text {ref }}$ and the initial condition defined in Table 1 , and produce the corresponding reference solution $\left(u_{h, \text { ref }}, w_{h, \text { ref }}\right)=\left(u_{h}, w_{h}\right)\left(\theta_{\text {ref }}\right)$ - namely, with (3.5). The associated measurements are made of a sampling of the solution at 9 equally spaced internal points in the segment, and at all times, with an additive Gaussian noise of standard deviation $\sigma_{\chi}$ generated independently at each sampling point, and to be adjusted consistently with $Q_{Z}$, see [5] for a related discussion. Then, we numerically solve ${ }^{6}$ the complete estimation problem associated with the criterion (4.1), which provides the estimated quantities $\left(\xi_{\star}, \theta_{\star}\right)$ and the corresponding solution $\left(u_{h \star}, w_{h \star}\right)=\left(u_{h}, w_{h}\right)\left(\xi_{\star}, \theta_{\star}\right)$. Note that we do not expect to exactly recover $\left(\xi_{\star}, \theta_{\star}\right)=\left(\xi_{\text {ref }}, \theta_{\text {ref }}\right)$ due to, in particular 1 - the added measurement noise, and 2- the a priori values $\left(\xi_{0}, \theta_{0}\right) \neq\left(\xi_{\text {ref }}, \theta_{\text {ref }}\right)$ used in $(3.5)$, hence we will consider the reference estimation errors

$$
\tau_{\xi}=\frac{\left\|\xi_{\star}-\xi_{\mathrm{ref}}\right\|_{Q_{\xi}}}{\left\|\xi_{\mathrm{ref}}\right\|_{Q_{\xi}}}, \quad \tau_{\theta}=\frac{\left\|\theta_{\star}-\theta_{\mathrm{ref}}\right\|_{Q_{\theta}}}{\left\|\theta_{\mathrm{ref}}\right\|_{Q_{\theta}}}, \quad \tau_{u}=\frac{\left\|u_{h \star}-u_{h, \mathrm{ref}}\right\|_{L_{\Delta t}^{2}(0, T ; V)}}{\left\|u_{h, \mathrm{ref}}\right\|_{L_{\Delta t}^{2}(0, T ; V)}},
$$

where $\xi_{\text {ref }}$ is defined by projecting the initial condition $u_{h}^{0}$ onto the selected eigenspace, viz.

$$
\xi_{\text {ref }}^{(i)}=\left(u_{h}^{0}, \psi_{i}\right)_{V}, \quad 1 \leq i \leq N_{\text {modes }}
$$

With the estimation setup as fully specified in Table 3, we obtain the reference errors listed in Table 4. Despite the fact that the a priori parameter vector $\theta_{0}$ used in the criterion significantly differs from the reference $\theta_{\text {ref }}$, these small errors estimation errors show that the parameter estimation problem is well-identifiable, including in the critical amplitude case $A=A_{c}$.

When numerically solving the reduced estimation problem associated with the criterion (4.6), we compare the resulting estimation with the reference by computing as indicators

$$
\eta_{\xi}(l)=\frac{\left\|\xi_{\star}^{l}-\xi_{\star}\right\|_{Q_{\xi}}}{\left\|\xi_{\star}\right\|_{Q_{\xi}}}, \quad \eta_{\theta}(l)=\frac{\left\|\theta_{\star}^{l}-\theta_{\star}\right\|_{Q_{\theta}}}{\left\|\theta_{\star}\right\|_{Q_{\theta}}}, \quad \eta_{u}(l)=\frac{\left\|u_{\star}^{l}-u_{h_{\star}}\right\|_{L_{\Delta t}^{2}(0, T ; V)}}{\left\|u_{h \star}\right\|_{L_{\Delta t}^{2}(0, T ; V)}} .
$$

\footnotetext{
${ }^{6}$ In practice, we use the gradient descent algorithm provided in the Matlab optimization toolbox by the function fminunc, a subspace trust-region method based on the interior-reflective Newton method.
} 

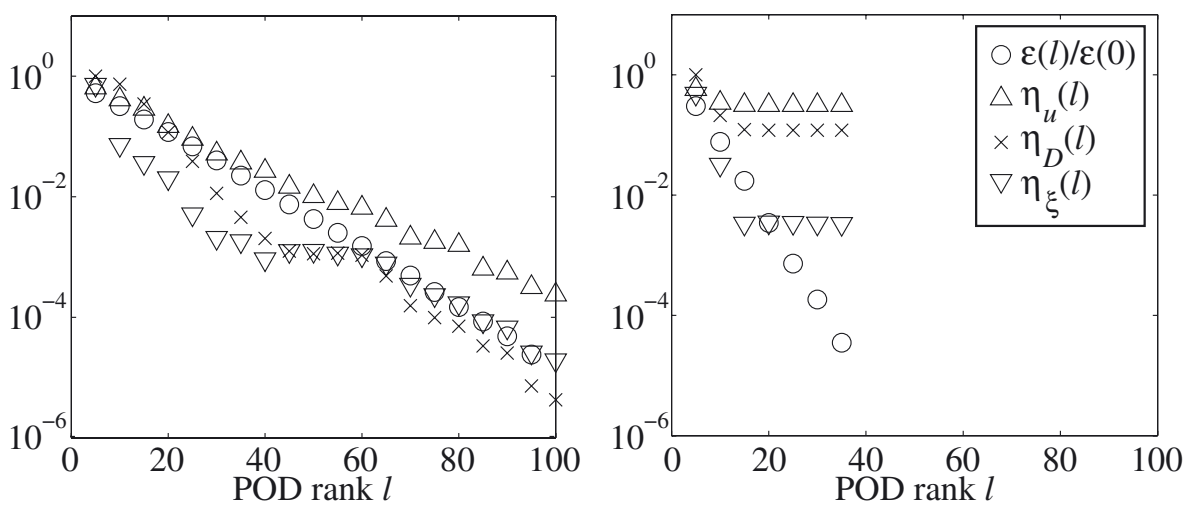

Figure 6. Error indicators vs. POD rank for multi-POD estimation (left) compared to standard POD estimation (right)
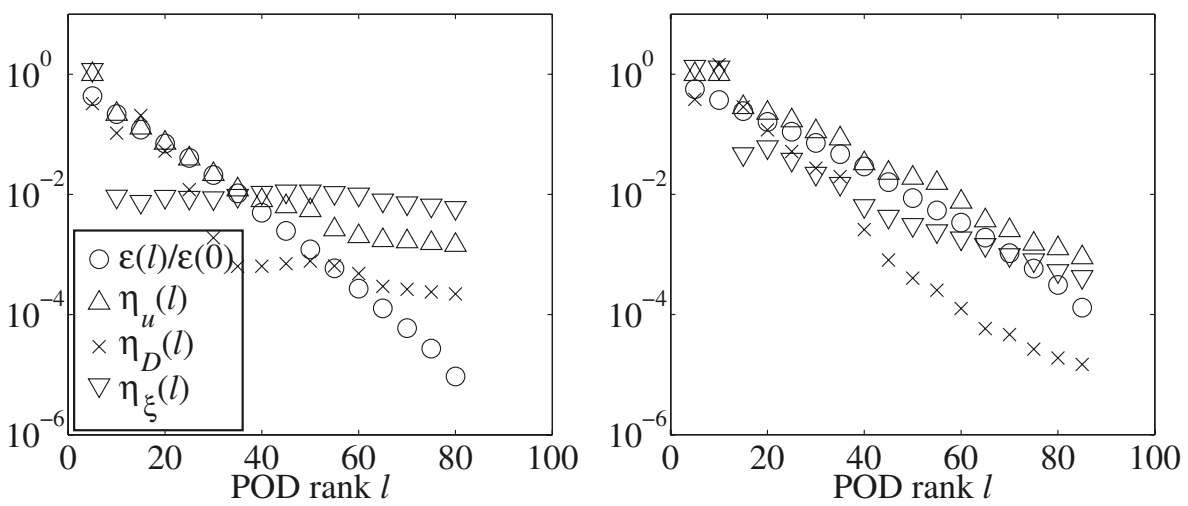

Figure 7. Error indicators vs. POD rank for multi-POD estimation at critical amplitude $A_{c}$ in reduced box $\mathcal{B}=[0.875 \bar{\theta}, 1.125 \bar{\theta}]^{2}$ (left) and for $Q_{2}$ multi-POD estimation in full box (right).

In the case of nominal amplitude, the results of the multi-POD estimation are plotted in Figure 6 and compared with estimation results obtained with the estimation based on standard POD construction. These error plots are in complete agreement with the above-discussed parametric assessments, recall Figure 2. In particular a convergence of $1 \%$ is achieved in all estimation indicators for roughly 45 modes in the multi-POD family, whereas the standard POD estimation fails to converge below $10 \%$.

Likewise, as in the parametric study, convergence difficulties are encountered for the reduced estimation in the critical amplitude case, and this requires the use of a reduced size parameter box for first-order interpolation, or of a higher-order - here quadratic - method in the same box. Note that, for the reduced box estimation, we changed the reference parameter vector to $\theta_{\text {ref }}=(1.05,0.9) \bar{\theta}$ in order for the parameter box to still feature both propagative and diffusive solutions, and then the reference estimation with the complete model converged in 35 iterations with $\left(\tau_{\xi}, \tau_{\theta}, \tau_{u}\right)=(0.92,0.22,0.34)$ in percent. The complete multi-POD estimation assessment results are plotted in Figure 7, again in very good agreement with the errors shown in Figure 5.

\subsection{Sequential estimation for a beating heart model with clinical data}

In this section, we demonstrate another application of estimation based on our proposed POD construction in a very different context. Namely, we consider the beating heart mechanical model described and analyzed in $[8,23]$ 
- already used to construct a test problem in [7] - and we will employ a sequential estimation strategy $[17,19]$ to estimate some uncertain quantities in a real case based on actual clinical measurements already considered in [5]. Therefore, this corresponds to a more complex estimation example - in many respects, including the model formulation, the data and the estimation procedure - than the previous case, and we will endeavor to present the various key ingredients in a compact manner, referring to previous papers for more details.

\subsubsection{Model summary}

The main equation is given by the so-called principle of virtual work, namely, the variational formulation

$$
\frac{\mathrm{d}}{\mathrm{d} t}(\underline{u}, \underline{v})+\mathcal{P}_{\mathrm{int}}\left(\underline{y}, \underline{u}, \iota_{c} ; \underline{v}\right)=\mathcal{P}_{\operatorname{ext}}(P, \underline{y} ; \underline{v}), \quad \forall \underline{v} \in V,
$$

where $\mathcal{P}_{\text {int }}$ and $\mathcal{P}_{\text {ext }}$ denote linear forms applying on the test field $\underline{v}$, these linear forms being themselves functions of the variables placed before the semicolons. These include $\iota_{c}$ in $\mathcal{P}_{\text {int }}$ which gathers a set of fields representing at each point the state of the contractile part of the behavior. The quantity $P$ in $\mathcal{P}_{\text {ext }}$ collects several scalar values representing the blood pressures in the main cardiovascular cavities. In the case considered here, these pressures are measured in the experimental protocol, hence the measured values are prescribed in the model equations. Alternatively, when pressure data are not available these quantities can be modeled using various strategies, see [23] and references therein. In addition, to close the dynamical system here, we have the displacement-velocity equation

$$
\frac{\mathrm{d} \underline{y}}{\mathrm{~d} t}=\underline{u},
$$

and some active dynamical constitutive equations modeling the evolution of the contractile variables

$$
\frac{\mathrm{d} \iota_{c}}{\mathrm{~d} t}=f_{c}\left(\iota_{c}, \underline{y}, U, \theta\right),
$$

where $U$ represents a given time-dependent activation field of electrophysiological nature and $\theta$ a set of socalled contractility parameters to be further discussed. We note the formal similarity of this system of equations (4.10)-(4.12) with the FitzHugh-Nagumo system (3.1)-(3.2), although of course here the conjunction of (4.10) and (4.11) make the dynamics essentially second-order in time.

Concerning discretization procedures, we consider a $P_{1}$-Lagrange finite element spatial discretization of displacements and velocities, and a mid-point time discretization of all dynamical equations, namely,

$$
\left\{\begin{array}{l}
\left(\frac{\underline{u}_{h}^{n+1}-\underline{u}_{h}^{n}}{\Delta t}, \underline{v}_{h}\right)+\mathcal{P}_{\operatorname{int}}\left(\underline{y}_{h}^{n+\frac{1}{2}}, \underline{u}_{h}^{n+\frac{1}{2}}, \iota_{c}^{n+\frac{1}{2}} ; \underline{v}_{h}\right)=\mathcal{P}_{\mathrm{ext}}\left(P^{n+\frac{1}{2}}, \underline{y}_{h}^{n+\frac{1}{2}} ; \underline{v}_{h}\right), \quad \forall \underline{v}_{h} \in V_{h} \\
\frac{\underline{y}_{h}^{n+1}-\underline{y}_{h}^{n}}{\Delta t}=\underline{u}_{h}^{n+\frac{1}{2}} \\
\frac{\iota_{c}^{n+1}-\iota_{c}^{n}}{\Delta t}=f_{c}\left(\iota_{c}^{n+\frac{1}{2}}, \underline{y}_{h}^{n+\frac{1}{2}}, U^{n+\frac{1}{2}}, \theta\right)
\end{array}\right.
$$

using for compactness the notation convention

$$
(.)^{n+\frac{1}{2}}=\frac{(.)^{n}+(.)^{n+1}}{2} .
$$

The Galerkin reduction is then obtained by specializing these equations to displacements and velocities in the subspace $V^{l}$, and likewise of course for the corresponding test functions. We denote the resulting solutions by $\left(\underline{y}^{l, n}, \underline{u}^{l, n}, \iota_{c}^{l, n}\right)$. We point out that we do not consider the internal variable $\iota_{c}$ in the POD construction, namely, only displacement snapshots are used to construct the POD basis as in [7]. Therefore, the internal variable dynamics equation

$$
\frac{\iota_{c}^{l, n+1}-\iota_{c}^{l, n}}{\Delta t}=f_{c}\left(\iota_{c}^{l, n+\frac{1}{2}}, \underline{y}^{l, n+\frac{1}{2}}, U^{n+\frac{1}{2}}, \theta\right)
$$


is solved exactly - at each numerical integration point - as in the complete model, albeit with the displacements in the reduced space. Nevertheless, this is not detrimental to computational effectiveness, since these internal variables are eliminated during the assembling process, which yields a displacement-only algebraic system to be solved.

\subsubsection{Sequential estimation procedure}

In contrast to the previous section, we will here perform joint state-parameter estimation by a sequential methodology, namely, the so-called Reduced-Order Unscented Kalman Filtering (RO-UKF) method proposed in [17] (see also [21]), an extension of classical Kalman filtering designed for non-linear systems. Compared to Extended Kalman Filtering (EKF), Unscented Kalman Filtering (UKF, see [14]) does not require to compute tangent operators for the dynamics and the measurements, and provides more accuracy due to second-order approximation of the mean of a probabilistic variable propagated by nonlinear operators.

Let us start by briefly summarizing the UKF principles, using a simplified model without internal variables for the procedure to be applicable as is, while the extension required to consider internal variables will be introduced in a second stage. We thus consider, instead of (4.13) and directly in reduced form,

$$
\left\{\begin{array}{l}
\left(\frac{\underline{u}^{l, n+1}-\underline{u}^{l, n}}{\Delta t}, \underline{v}\right)+\overline{\mathcal{P}}_{\mathrm{int}}\left(\underline{y}^{l, n+\frac{1}{2}}, \underline{u}^{l, n+\frac{1}{2}}, t^{n+\frac{1}{2}}, \theta ; \underline{v}\right) \\
=\overline{\mathcal{P}}_{\mathrm{ext}}\left(\underline{y}^{l, n+\frac{1}{2}}, t^{n+\frac{1}{2}} ; \underline{v}\right), \quad \forall \underline{v} \in V^{l} \\
\frac{\underline{y}^{l, n+1}-\underline{y}^{l, n}}{\Delta t}=\underline{u}^{l, n+\frac{1}{2}} .
\end{array}\right.
$$

Also, to fix the ideas we assume that the measurements are given by a relation of the form

$$
Z^{n}=H\left(\underline{y}\left(t^{n}\right)\right)+\chi_{\Delta t},
$$

where $Z^{n}$ is a finite-dimensional vector, and $\chi_{\Delta t}$ a Gaussian noise of covariance operator $Q_{Z}^{-1} / \Delta t$, with the usual scaling associated with the time sampling rate $\Delta t[18]$.

For the purpose of estimation by filtering, the state variable associated with (4.14) is equivalent to $\left(\alpha^{l, n}, \beta^{l, n}\right)$, where $\alpha^{l, n}$ and $\beta^{l, n}$ respectively concatenate the coefficients of the decompositions of $\underline{y}^{l, n}$ and $\underline{u}^{l, n}$ in the POD basis. In our case, since we are primarily interested in estimating the parameter vector $\theta$ - for which we need to perform joint state-parameter estimation - we classically introduce the augmented state given by

$$
X^{n}=\left(\alpha^{l, n} \beta^{l, n} \theta\right)^{\top},
$$

and we can then see (4.14) as a set of relations allowing to infer $X^{n+1}$ from $X^{n}$, with the particular rule $\theta^{n+1}=\theta^{n}=\theta$. Namely, we have a discrete model summarized by

$$
X^{n+1}=A_{n+1 \mid n}\left(X^{n}\right), \quad X^{0}=X_{0}+\zeta_{X},
$$

incorporating an uncertainty represented by $\zeta_{X}$ in the initial condition, hence including the parameter values. We will thus construct the time-dependent estimation $\hat{X}^{n}$ in a probabilistic, Bayesian, framework which - based on the available measurements $Z^{n}$ - will provide an estimation of the state variable trajectory, and simultaneously of the parameter vector $\theta$. The parameter estimation component $\hat{\theta}^{n}$ will thus evolve over time, but of course should rapidly converge to the reference value when the procedure is effective, which requires some adequate observability conditions, in particular.

In the context of Kalman filtering, the recursive estimation is based on the Best Linear Unbiased Estimator (BLUE), see e.g. [24]. Namely, assuming that we have obtained an estimation $\hat{X}^{n,+}$ at time $t^{n}$ as a probabilistic variable given all the observations up to $Z^{n}$, with mean $\hat{X}^{n}$ and covariance $\Gamma^{n}$, we first infer a prediction at step $n+1$ by directly applying the dynamics (4.14), which gives a new probabilistic variable $\hat{X}^{n+1,-}$. Then, 
taking into account the new data $Z^{n+1}$ the BLUE rule gives, as approximations of the new mean and covariance given all the observations up to $Z^{n+1}$,

$$
\left\{\begin{array}{l}
\hat{X}^{n+1}=\mathrm{E}\left(\hat{X}^{n+1,-}\right)+\operatorname{Cov}\left(\hat{X}^{n+1,-}, Z^{n+1}\right) \operatorname{Cov}\left(Z^{n+1}\right)^{-1}\left(Z^{n+1}-\mathrm{E}\left(H\left(\hat{X}^{n+1,-}\right)\right)\right) \\
\Gamma^{n+1}=\operatorname{Cov}\left(\hat{X}^{n+1,-}\right)-\operatorname{Cov}\left(\hat{X}^{n+1,-}, Z^{n+1}\right) \operatorname{Cov}\left(Z^{n+1}\right)^{-1} \operatorname{Cov}\left(Z^{n+1}, \hat{X}^{n+1,-}\right)
\end{array}\right.
$$

where all mean and covariance operators should be understood as with observations given up to $Z^{n}$. Of course, with the nonlinear operators employed in the dynamics and measurement equations, these quantities cannot be exactly computed in general. Unlike for EKF in which an approximation is obtained using the linearized forms of these operators, the UKF approximation is based on a representation of probability distributions based on so-called sampling points. At step $n$, such sampling points are defined as $p$ vectors $\left(\hat{X}_{i}^{n,+}\right)_{i=1}^{p}$ of empirical mean and covariance exactly equal to $\hat{X}^{n}$ and $\Gamma^{n}$, namely,

$$
\left\{\begin{array}{l}
\mathrm{E}_{\alpha}\left(\hat{X}_{*}^{n,+}\right)=\sum_{i=1}^{p} \alpha_{i} \hat{X}_{i}^{n,+}=\hat{X}^{n} \\
\operatorname{Cov}_{\alpha}\left(\hat{X}_{*}^{n,+}\right)=\mathrm{E}_{\alpha}\left(\left(\hat{X}_{*}^{n,+}-\mathrm{E}_{\alpha}\left(\hat{X}_{*}^{n,+}\right)\right)\left(\hat{X}_{*}^{n,+}-\mathrm{E}_{\alpha}\left(\hat{X}_{*}^{n,+}\right)\right)^{\top}\right)=\Gamma^{n}
\end{array}\right.
$$

where $\left(\alpha_{i}\right)_{i=1}^{p}$ denote some well-chosen scalar weights, see e.g. [17] for adequate choices and associated sampling constructions. Applying the dynamics operator on each individual sampling point, we directly infer the propagated sampling points $\left(\hat{X}_{i}^{n+1,-}\right)_{i=1}^{p}$ which represent the distribution of $\hat{X}^{n+1,-}$, and which are in turn used to approximate the means and covariances in (4.16) by their empirical counterparts, viz.

$$
\left\{\begin{aligned}
\hat{X}^{n+1}=\mathrm{E}_{\alpha}\left(\hat{X}_{*}^{n+1,-}\right)+\operatorname{Cov}_{\alpha}\left(\hat{X}_{*}^{n+1,-}, H\left(\hat{X}_{*}^{n+1,-}\right)\right) & \\
& \times\left[\operatorname{Cov}\left(\chi_{\Delta t}\right)+\operatorname{Cov}_{\alpha}\left(H\left(\hat{X}_{*}^{n+1,-}\right)\right)\right]^{-1}\left(Z^{n+1}-\mathrm{E}_{\alpha}\left(H\left(\hat{X}_{*}^{n+1,-}\right)\right)\right) \\
\Gamma^{n+1}=\operatorname{Cov}_{\alpha}\left(\hat{X}_{*}^{n+1,-}\right)-\operatorname{Cov}_{\alpha}\left(\hat{X}_{*}^{n+1,-}, H\left(\hat{X}_{*}^{n+1,-}\right)\right) & \\
\times & {\left[\operatorname{Cov}\left(\chi_{\Delta t}\right)+\operatorname{Cov}_{\alpha}\left(H\left(\hat{X}_{*}^{n+1,-}\right)\right)\right]^{-1} \operatorname{Cov}_{\alpha}\left(\hat{X}_{*}^{n+1,-}, H\left(\hat{X}_{*}^{n+1,-}\right)\right) }
\end{aligned}\right.
$$

Note that the covariance matrices $\Gamma^{n}$ computed in this framework have the size of the (augmented) state variable $X$ and are full matrices, hence this provides a tractable method for our model problem (4.14) only because we use POD reduction and estimate a finite number of scalar parameters. However, when considering the original problem (4.13) we also need to deal with the internal variables $\iota_{c}$, which are not directly concerned by POD reduction as explained above. Therefore, we need to resort to an important generalization, namely reduced-order UKF (RO-UKF). This means that the uncertainty is assumed to be restricted to a subspace of the state space, in which case the covariance matrices can be factorized in the form

$$
\Gamma^{n}=L^{n}\left(U^{n}\right)^{-1}\left(L^{n}\right)^{\top},
$$

where $U^{n}$ has the size of the uncertainty subspace. Then, from sampling points $\left(R_{i}^{n}\right)_{i=1}^{p}$ constructed in the reduced size subspace - which of course represents the major benefit of considering reduced uncertainty - with zero mean and empirical covariance $\left(U^{n}\right)^{-1}$, we easily infer that the $p$ vectors

$$
\hat{X}_{i}^{n,+}=\hat{X}^{n}+L^{n} R_{i}^{n},
$$

are sampling points with $\hat{X}^{n}$ and $\Gamma^{n}$ as empirical mean and covariance, respectively. Moreover, the RO-UKF method is specifically designed to obtain from (4.18) a similarly factorized expression of $\Gamma^{n+1}$, see [17].

In our case, we will assume that the original modeling uncertainty is restricted to the displacement initial condition and to the parameter values. This is substantiated by the fact that internal variables are in a welldefined state before the electrical activation starts. Accordingly, we set up $\Gamma^{0}$ in the factorized form (4.19) where the dimension of $U^{0}$ is that of the POD space added to the number of parameters to be estimated. Then $L^{0}$ gives zero covariance on all other state variables, namely, velocities and internal variables $\tau_{c}$. 

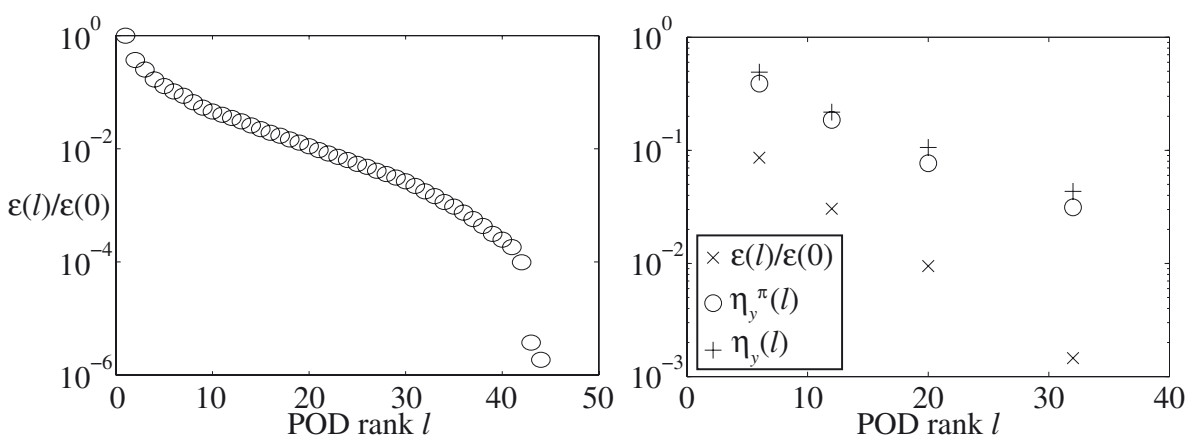

FiguRe 8. Relative POD remainder vs. POD rank for heart model (left) and comparison with displacement error and projection error indicators (right).

Moreover, we will need to resort to another generalization of the method, because the data cannot be associated with an observation operator in the form (4.15) per se. In our case, we will use as measurements a sequence of MR images, segmented in order to extract the contours of the left ventricle of the heart. In order to compare the model with the data, we thus introduce a so-called discrepancy operator $D(y, t)$ which computes distance fields from the points of the current model contour to the corresponding segmented image contour [19]. Hence, this operator is an extension of the so-called innovation term in data assimilation, which would be standardly given by an expression of the form " $z(t)-H(\underline{y})$ ". The straightforward extension of (4.18) is then

$$
\left\{\begin{aligned}
\hat{X}^{n+1}= & \mathrm{E}_{\alpha}\left(\hat{X}_{*}^{n+1,-}\right)-\operatorname{Cov}_{\alpha}\left(\hat{X}_{*}^{n+1,-}, D\left(\hat{X}_{*}^{n+1,-}, t^{n+1}\right)\right) \\
& \times\left[\operatorname{Cov}\left(\chi_{\Delta t}\right)+\operatorname{Cov}_{\alpha}\left(D\left(\hat{X}_{*}^{n+1,-}, t^{n+1}\right)\right)\right]^{-1} \mathrm{E}_{\alpha}\left(D\left(\hat{X}_{*}^{n+1,-}, t^{n+1}\right)\right) \\
\Gamma^{n+1}= & \operatorname{Cov}_{\alpha}\left(\hat{X}^{n+1,-}\right)-\operatorname{Cov}_{\alpha}\left(\hat{X}_{*}^{n+1,-}, D\left(\hat{X}_{*}^{n+1,-}, t^{n+1}\right)\right) \\
& \times\left[\operatorname{Cov}\left(\chi_{\Delta t}\right)+\operatorname{Cov}_{\alpha}\left(D\left(\hat{X}_{*}^{n+1,-}, t^{n+1}\right)\right)\right]^{-1} \operatorname{Cov}_{\alpha}\left(\hat{X}_{*}^{n+1,-}, D\left(\hat{X}_{*}^{n+1,-}, t^{n+1}\right)\right)^{\top}
\end{aligned}\right.
$$

\subsubsection{Estimation results with real image data}

We now endeavor to apply the above sequential estimation strategy in a real case where actual MR (magnetic resonance) images have been acquired on an infarcted pig heart [5]. In this case, we will estimate some values of the contractility parameter that represents the ability of the myocardium to contract under a given electrical activation [8]. First, the infarcted region has been manually delineated in the so-called late enhancement MR images which - together with the healthy myocardium - defines two distinct regions within each of which we will estimate constant contractility values. The data used for estimation purposes consists of so-called Cine-MR images, namely, in this case 24 successive image sets - of 13 cross-sections each - taken along the heart beat, in which the contours of the left ventricle have been manually segmented. These segmented surfaces provide the reference for a discrepancy operator based on the distance computed between the corresponding model contours and these surfaces [19].

Scaling the contractility values to one for a reference healthy behavior, we thus consider the 2D parameter box $\mathcal{B}=[0.75,1.25] \times[0,1]$ where the first value is associated with the healthy region. Accordingly, we simulated four trajectories for $Q_{1}$ multi-POD generation, each of them covering a complete heart beat period (duration $0.6 \mathrm{~s}$ ). The numerical system considered has about 25000 displacement degrees of freedom, and time discretization is performed with a mid-point Newmark scheme (600 time steps of $1 \mathrm{~ms}$ each for every simulation), see [23] for more details on the discretization procedures. In this case, due to the size of the state vector we only stored 12 equally-spaced snapshots for each trajectory. The linearized stiffness operator associated with the reference configuration was used as scalar product in the POD computation, namely, to define the scalar product of $V$. The resulting POD remainder values $\varepsilon(l)$ are shown in Figure 8-left. 
TABLE 5. Error indicators for estimated parameters.

\begin{tabular}{|l|cccc|}
\hline$l$ & 6 & 12 & 20 & 32 \\
\hline$\eta_{\theta_{1}}(l)(\%)$ & 9.9 & 5.7 & 5.4 & 2.6 \\
$\eta_{\theta_{2}}(l)(\%)$ & 30.7 & 0.6 & 4.4 & 3.6 \\
\hline
\end{tabular}

We then apply the above-described RO-UKF estimation method with this multi-POD family, for a varying number of POD members in order to assess the convergence of the estimation. In the absence of any ground truth - recall that we are considering real data - we use the estimation obtained with a rather large number of POD members, namely $l=44$ - corresponding to a very small remainder in Figure 8-left - as a reference solution for computing error indicators. More specifically, denoting by $\underline{\hat{y}}^{\text {ref }}$ and $\hat{\theta}^{\text {ref }}$ the reference $(l=44)$ estimated trajectories for the displacement field and contractility parameters, respectively, we consider the indicators

$$
\eta_{y}(l)=\frac{\left\|\underline{\hat{y}}^{l}-\underline{\hat{y}}^{\mathrm{ref}}\right\|_{L_{\Delta t_{s}}^{2}(0, T ; V)}}{\left\|\underline{\hat{y}}^{\mathrm{ref}}\right\|_{L_{\Delta t_{s}}^{2}(0, T ; V)}}, \quad \eta_{\theta_{i}}(l)=\frac{\left|\hat{\theta}_{i}^{l}(T)-\hat{\theta}_{i}^{\mathrm{ref}}(T)\right|}{\left|\hat{\theta}_{i}^{\mathrm{ref}}(T)\right|},
$$

where the approximate $L_{\Delta t_{s}}^{2}(0, T ; V)$-norm is computed using only the time steps retained in the snapshots employed in the multi-POD generation - in order to have quantities directly comparable to the POD remain$\operatorname{der} \varepsilon(l)$.

With the initial covariance set to $10^{-7} \mathrm{Id}_{p}$ for the POD coefficients - which can be shown to scale to a small mean energy with respect to which we checked that the estimation result was not sensitive - and to $2 \operatorname{Id}_{2}$ for the parameters, we obtained the results displayed in Figure 8-right for the behavior of the displacement indicator compared with the POD remainder and with the projection error indicator

$$
\eta_{y}^{\pi}(l)=\frac{\left\|\underline{\hat{y}}^{\mathrm{ref}}-\pi_{V}^{l} \underline{\hat{y}}^{\mathrm{ref}}\right\|_{L_{\Delta t_{s}}^{2}(0, T ; V)} .}{\left\|\underline{\hat{y}}^{\mathrm{ref}}\right\|_{L_{\Delta t_{s}}^{2}(0, T ; V)}}
$$

We observe that the reduced observer with 32 modes reaches a very satisfactory performance of $4 \%$ relative error in the displacement indicator $\eta_{y}$. We also note that this error indicator is very close to the reference projection error for all values of $l$, albeit both being significantly larger than the POD remainder, which may be attributed to the coarseness of the parameter box. Table 5 lists the parameter indicator values, and we can see that the convergence levels achieved are comparable to - and as satisfactory, indeed, as - those obtained for the displacement fields. It should also be pointed out that the estimated contractility values,

$$
\hat{\theta}_{1}^{\mathrm{ref}}(T)=0.77, \quad \hat{\theta}_{2}^{\mathrm{ref}}(T)=0.38
$$

very markedly reveal the infarct impact in the second region.

Finally, we display in Figure 9 the simulated observer contours compared with MR images at two stages, namely, before and at the end of the contraction (systole), in a central cross-section representative of the whole behavior. The zoom views show that the observer corresponding to $l=32$ is very close to the reference contour, both of them being in excellent adequacy with the imaged contraction motion, indeed, including for the right ventricle wall (upper-left part of the cross-sections and Zoom 2) in which the wall was not segmented, hence not used in the filtering corrections.

Concerning the computational costs, in the off-line stage the four parametric solutions are computed in parallel, and the POD construction induces only a very slight overhead. In the estimation stage, RO-UKF estimation necessitates - at each time step - a one-step-forward simulation for each state associated with a sampling point. In our case, with 2 parameters to be estimated we need $l+3$ such sampling points. These forward simulations are completely independent of each other, so they can be performed in parallel, and for each of them POD reduction allows to roughly reduce the computation time by $50 \%$, with the full assembling 


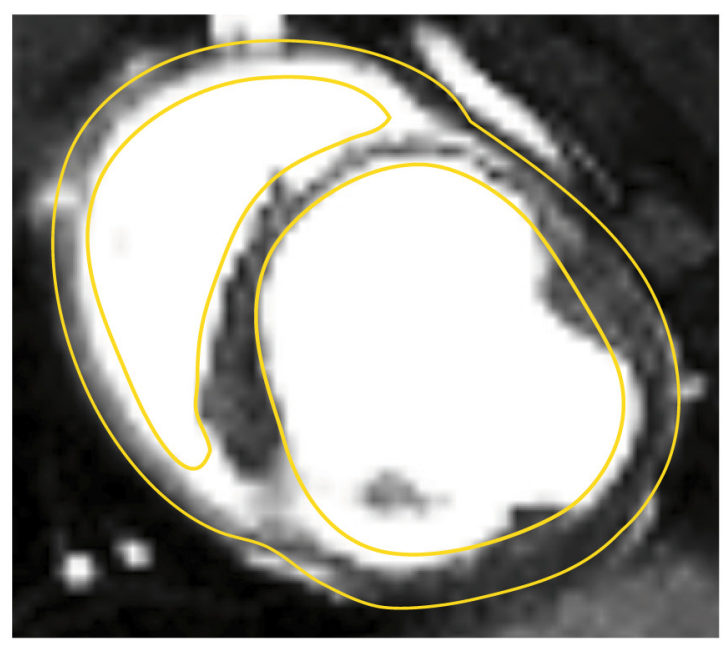

$$
\mathrm{t}=0 \mathrm{~ms}
$$

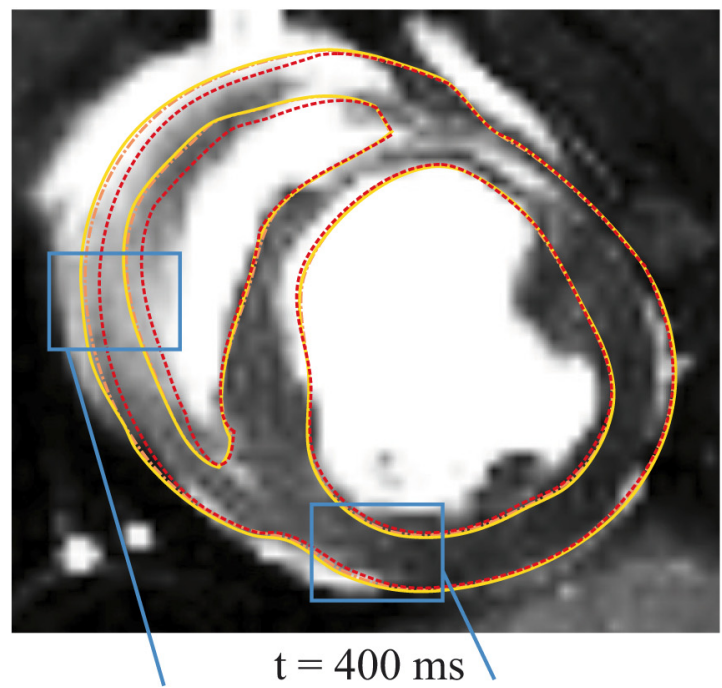

Zoom 2

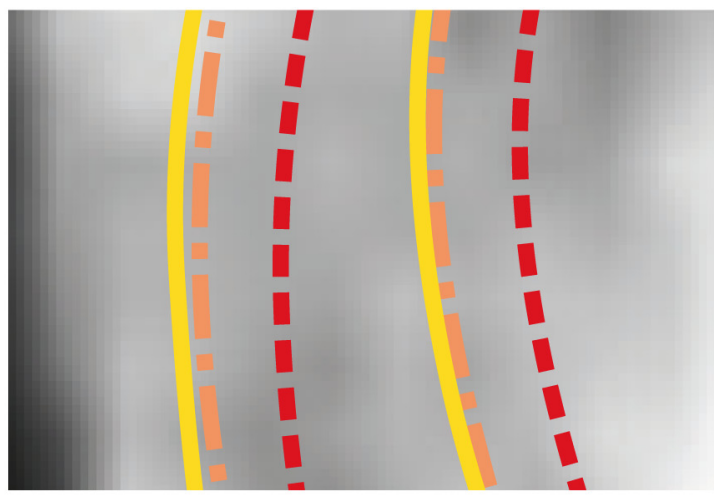

Zoom 1

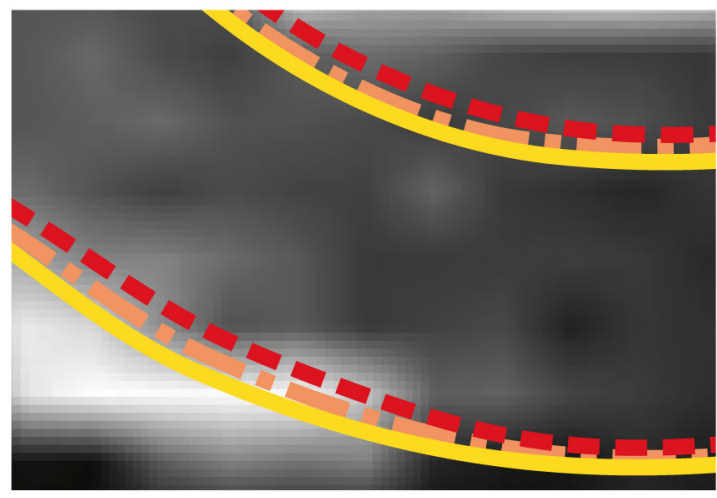

Zoom 2

Figure 9. Comparison of observer contours with MR images at two stages for $l=$ $12,32,44$ (top) and zoomed views (bottom).

process still being performed. Subsequently, the distances to segmented contours are computed for each particle - also fully in parallel - with a cost comparable to assembling, hence the total computation time for each sampling point is close to that of an unreduced direct simulation. Finally, estimation overheads at each time step are quite modest, so the total estimation run can be performed in roughly the same time as a single unreduced direct simulation, of course on a parallel machine with a sufficient number of nodes.

\section{Concluding Remarks}

We have derived some new a priori error estimates for POD approximations of parameter-dependent solutions of parabolic problems, without any specific assumption on the form of the parameter dependence. These error estimates suggest - and substantiate, indeed - a natural POD construction strategy, consisting in concatenating the snapshots of the solutions associated with the interpolation "nodes" considered in the parameter space. Of course, the relevant parameter nodes may vary depending on the actual parameter vector for which a solution 
is sought, according to the geometric subdivision - or "mesh" - considered in the parameter space. We then also resorted to the same application examples as in [7] - chosen within cardiac modeling - to illustrate and assess our strategy, namely, the 1D FitzHugh-Nagumo electrophysiology model, and a complete 3D nonlinear mechanical model of a beating heart. In the case of the FitzHugh-Nagumo model we numerically computed the approximation errors made with the POD reduction, and found these numerical errors to be consistent with the a priori error estimates derived, even though the nonlinearity contained in the model places it outside of the analysis scope. In addition, each of the two models was successfully used to demonstrate a different estimation strategy: adjoint-based variational estimation for the FitzHugh-Nagumo model with synthetic measurements, and sequential estimation for the beating heart model in a complex "patient-specific" context with actual image data used as measurements. In addition, we have observed in these numerical examples that a single element in the parameter space provides rather accurate results even with bilinear interpolation for substantial parameter variations, except when the solution is very irregular with respect to this parameter dependence.

In order to further put this strategy into perspective, we point out that in [5] sequential estimation was also performed in the same beating heart case, albeit without resorting to POD reduced-order modeling. Instead, a reduced-order sequential estimation strategy was used - namely, RO-UKF - in which a first stage Luenberger observer estimation was applied to reduce - in essence - the uncertainty to the parameter space $[17,19]$. The results obtained here are consistent with those of [5], but in this previous work a much larger number of contractility parameters were estimated, i.e. up to 17 scalar values. These parameters were associated with a more detailed anatomical subdivision, thus allowing to effectively identify the location of the infarct via the estimation itself. In the present estimation setup, the limiting factor concerning the number of parameters lies in the number of solutions to be pre-computed in order to construct the POD family. Nevertheless, while the estimation strategy of [5] is much more powerful in this respect, it requires a Luenberger observer tailored to the model at hand, whereas the POD-based method presented herein can be seen as a "black box" approach that can be directly applied to a large class of problems.

Concerning the "curse of dimensionality" faced in our approach due to computational complexity increasing exponentially with the dimension of the parameter space, we should also mention that some alternative interpolation strategies are available in order to more effectively handle parameter spaces of larger dimensions, as is the purpose of sparse grid interpolation [25], in particular. Nevertheless, for still higher parameter dimensions other approaches should be investigated, such as greedy algorithms [4,13], see also [12]. Finally, whatever approximation approach is considered, a posteriori error estimates are a natural - very important - perspective once an a priori error analysis has been achieved, see e.g. [26] and references therein.

\section{REFERENCES}

[1] D. Amsallem and C. Farhat, An online method for interpolating linear parametric reduced-order models. SIAM J. Sci. Comput. 33 (2011) 2169.

[2] H.T. Banks, M.L. Joyner, B. Winchesky and W.P. Winfree, Nondestructive evaluation using a reduced-order computational methodology. Inverse Problems 16 (2000) 1-17.

[3] G. Berkooz, P. Holmes and J.L. Lumley, The proper orthogonal decomposition in the analysis of turbulent flows. Annu. Rev. Fluid Mech. 25 (1993) 539-575.

[4] A. Buffa, Y. Maday, A.T. Patera, C. Prud'homme and G. Turinici, A priori convergence of the greedy algorithm for the parametrized reduced basis method. ESAIM: M2AN 46 (2012) 595-603.

[5] R. Chabiniok, P. Moireau, P.-F. Lesault, A. Rahmouni, J.-F. Deux and D. Chapelle, Estimation of tissue contractility from cardiac cine-MRI using a biomechanical heart model. Biomech. Model. Mechanobiol. 11 (2012) 609-630.

[6] D. Chapelle and K.J. Bathe, The inf-sup test. Comput. Struct. 47 (1993) 537-545.

[7] D. Chapelle, A. Gariah and J. Sainte-Marie, Galerkin approximation with Proper Orthogonal Decomposition: new error estimates and illustrative examples. ESAIM: M2AN 46 (2012) 731-757.

[8] D. Chapelle, P. Le Tallec, P. Moireau and M. Sorine, An energy-preserving muscle tissue model: formulation and compatible discretizations. J. Multiscale Comput. Engrg. 10 (2012) 189-211.

[9] G. Chavent, Nonlinear Least Squares for Inverse Problems: Theoretical foundations and step-by-step guide for applications. Scientific Computation. Springer, New York (2009).

[10] P.G. Ciarlet and P.A. Raviart, General Lagrange and Hermite interpolation in $\mathbb{R}$ with applications to finite element methods. Arch. Rational Mech. Anal. 46 (1972) 177-199. 
[11] R. FitzHugh, Impulses and physiological states in theoretical models of nerve membrane. Biophys. J. 1 (1961) $445-466$.

[12] D. Galbally, K. Fidkowski, K. Willcox and O. Ghattas, Non-linear model reduction for uncertainty quantification in large-scale inverse problems. International J. Numer. Methods Engrg. 81 (2010) 1581-1608.

[13] B. Haasdonk, Convergence rates of the POD-greedy method. ESAIM: M2AN 47 (2012) 859-873.

[14] S. Julier, J. Uhlmann and H. Durrant-Whyte, A new method for the nonlinear transformation of means and covariances in filter and estimators. IEEE Trans. Automat. Contr. 45 (2000) 447-482.

[15] K. Kunisch and S. Volkwein, Galerkin proper orthogonal decomposition methods for a general equation in fluid dynamics. SIAM J. Numer. Anal. 40 (2002) 492-515.

[16] A. Manzoni, A. Quarteroni and G. Rozza, Shape optimization for viscous flows by reduced basis methods and free form deformation. Int. J. Numer. Methods in Fluids 70 (2012) 646-670.

[17] P. Moireau and D. Chapelle, Reduced-order Unscented Kalman Filtering with application to parameter identification in largedimensional systems. ESAIM: COCV 17 (2011) 380-405.

[18] P. Moireau, D. Chapelle and P. Le Tallec, Joint state and parameter estimation for distributed mechanical systems. Comput. Methods Appl. Mechanics Engrg. 197 (2008) 659-677.

[19] P. Moireau, D. Chapelle and P. Le Tallec, Filtering for distributed mechanical systems using position measurements: Perspectives in medical imaging. Inverse Problems 25 (2009) 035010.

[20] J. Nagumo, S. Arimoto and S. Yoshizawa, An active pulse transmission line simulating nerve axon. Proc. of IRE 50 (1962) 2061-2070.

[21] D.-T. Pham, J. Verron and L. Gourdeau, Filtres de Kalman singuliers évolutifs pour l'assimilation de données en océanographie. C.R. l'Acad. Sci. - Series IIA 326 (1998) 255-260.

[22] C. Prud'homme, D.V. Rovas, K. Veroy, L. Machiels, Y. Maday, A.T. Patera and G. Turinici, Reliable real-time solution of parametrized partial differential equations: Reduced-basis output bound methods. J. Fluids Engrg. 124 (2002) 70-80.

[23] J. Sainte-Marie, D. Chapelle, R. Cimrman and M. Sorine, Modeling and estimation of the cardiac electromechanical activity. Comput. Struct. 84 (2006) 1743-1759.

[24] D. Simon, Optimal State Estimation: Kalman, $H^{\infty}$, and Nonlinear Approaches. Wiley-Interscience (2006).

[25] S.A. Smolyak. Quadrature and interpolation formulas for tensor products of certain classes of functions. Dokl. Akad. Nauk SSSR 4 (1963) 240-243.

[26] K. Veroy and A.T. Patera, Certified real-time solution of the parametrized steady incompressible navier-stokes equations. Internat. J. Numer. Methods Fluids 47 (2004) 773-788. 